\title{
Enhanced Photoluminescence of Sulfur-Bridged Organic Chromophores
}

\author{
Peter R. Christensen, ${ }^{\S}$ Jeffrey K. Nagle, ${ }^{\dagger}$ Aini Bhatti, ${ }^{\S}$ and Michael O. Wolf ${ }^{*}$, \\ ${ }^{\S}$ Department of Chemistry, University of British Columbia, Vancouver, BC, V6T 1Z1, Canada. \\ ${ }^{\dagger}$ Department of Chemistry, Bowdoin College, Brunswick, Maine, 04011, United States.
}

\section{Experimental Details}

\section{General}

Electronic absorption spectroscopy was performed on a Varian Cary 5000 spectrophotometer. Corrected emission measurements were performed on a PTI QuantaMaster 50 fluorimeter at room temperature in $\mathrm{CH}_{2} \mathrm{Cl}_{2}$ (unless otherwise specified). All solvents used for photochemical measurements, dichloromethane, cyclohexane, and acetonitrile were analytical HPLC grade, and used as received from Fisher Scientific. Samples were purged with argon for no less than $15 \mathrm{~min}$ before emission studies. Photoluminescence quantum yields $\left(\Phi_{\mathrm{f}}\right)$ for each compound were calculated according to equation $\mathrm{S} 1$, using 9-cyanoanthracene as a standard.

$$
\Phi_{x}=\left(\frac{A_{s}}{A_{x}}\right)\left(\frac{F_{x}}{F_{s}}\right)\left(\frac{n_{x}}{n_{s}}\right)^{2} \Phi_{s}
$$

equation S1.

$A$ is the absorption at the excitation wavelength for the standard (s) and the compound of interest (x), $F$ is the integrated area under the emission spectrum, $n$ is the refractive index of the solvent and $\Phi_{\mathrm{s}}$ is the known quantum yield for the standard in a given solvent $\left(\Phi_{9 \text {-cyanoanthracene }}=0.80\right.$ in methanol, excited at $364 \mathrm{~nm}$ ). ${ }^{1}$ The following excitation wavelengths were used for the compounds: for all $\mathbf{1 S O}_{\mathbf{n}}=335 \mathrm{~nm}$, $\mathbf{2 S O}_{\mathbf{n}}=375 \mathrm{~nm}, \mathbf{3 S O}_{\mathbf{n}}=304 \mathrm{~nm}$, and $\mathbf{4 S O}_{\mathbf{n}}=362 \mathrm{~nm}$. To correct for excitation intensity variation as a function of wavelength, the relative intensity of the excitation light at each wavelength was determined by integrating the excitation profile obtained with no sample present. The collected emission spectra $(F)$ for the samples were then scaled relative to the excitation intensity at $364 \mathrm{~nm}$ where the standard was excited.

To analyze the statistical significance of this method, and to ascertain the statistical significance of the observed increase in $\Phi_{\mathrm{f}}$ as a function of the bridging sulfur oxidation state, data was collected for each sample at three different concentrations for all compounds including 9-cyanoanthracene. The $\Phi_{\mathrm{f}}$ was calculated using equation S1 at each concentration and the resulting data was plotted against the known concentrations. The standard deviation in $\Phi_{\mathrm{f}}$ was calculated using the measured $\Phi_{\mathrm{f}}$ at different concentrations. 
Nuclear magnetic resonance (NMR) experiments were performed on either a 300, 400, or $600 \mathrm{MHz}$ Bruker spectrometer in deuterated chloroform $\left(\mathrm{CDCl}_{3}\right)$ or dichloromethane $\left(\mathrm{CD}_{2} \mathrm{Cl}_{2}\right)$. Compounds 1 and $\mathbf{2}$ were characterized by 1 -dimensional ${ }^{1} \mathrm{H}$ NMR only. The structures of compounds $\mathbf{3}$ and $\mathbf{4}$ were assigned using 2-dimensional NMR experiments, including COSY, ${ }^{1} \mathrm{H}^{13} \mathrm{C}$ HSQC, and HMBC.

Infrared spectroscopy (IR) was performed on an attenuated total reflection (ATR) crystal using a PerkinElmer Frontier FT-IR spectrometer.

Solid-state photoluminescence measurements were made on pre-cleaned quartz slides (sonicated 15 min each in methanol, chloroform, and again with methanol and allowed to dry in an oven for several hours), and $\sim 1 \mathrm{~cm}^{2}$ alumina TLC plates without added fluorescent indicator.

Equations S1-S3 were generated using open source web-based software, http://www.codecogs.com/latex/eqneditor.php.

\section{Synthetic Details}

\section{Synthesis of $1 \mathrm{~S}$}

To a solution of 2,2'-bithiophene $(363 \mathrm{mg}, 2.18 \mathrm{mmol})$ in dry tetrahydrofuran $(8.0 \mathrm{~mL})$ at $-78{ }^{\circ} \mathrm{C}$ under nitrogen was slowly added $n$-butyllithium $(1.6 \mathrm{M}$ in hexanes, $1.40 \mathrm{~mL}, 2.24 \mathrm{mmol})$. The mixture was stirred for $1 \mathrm{~h}$. To the resultant mixture was added drop-wise a solution of bis(phenylsulfonyl)sulfide (366 mg, $1.16 \mathrm{mmol})$ in dry tetrahydrofuran $(5.0 \mathrm{~mL})$ and the mixture was stirred overnight. The mixture was poured over water $(25 \mathrm{~mL})$ and extracted with diethylether $(3 \times 15 \mathrm{~mL})$. The organic layers were combined and dried over anhydrous magnesium sulfate, filtered, and concentrated under vacuum. Purification by column chromatography (hexanes) gave a yellow-white solid. Yield: $255 \mathrm{mg}, 65 \%$. ${ }^{1} \mathrm{H}$ $\operatorname{NMR}\left(400 \mathrm{MHz}, \mathrm{CD}_{2} \mathrm{Cl}_{2}\right): \delta 7.26(\mathrm{dd}, 2 \mathrm{H}, J=1.3,5.1 \mathrm{~Hz}), 7.17-7.15(\mathrm{~m}, 4 \mathrm{H}), 7.05(\mathrm{~d}, 2 \mathrm{H}, J=3.7 \mathrm{~Hz})$, $7.01(\mathrm{dd}, 2 \mathrm{H}, J=3.7,5.0 \mathrm{~Hz}$ ). HRMS (ESI): $\mathrm{m} / z$ calculated: 361.9386 , found 361.9389 .

\section{Synthesis of 1 SO}

To a solution of $1(129 \mathrm{mg}, 0.356 \mathrm{mmol})$ in dichloromethane $(6.0 \mathrm{~mL})$ at $0{ }^{\circ} \mathrm{C}$ was added metachloroperoxybenzoic acid $(70 \%, 89 \mathrm{mg}, 0.36 \mathrm{mmol})$ and the mixture was stirred for $15 \mathrm{~min}$. The reaction mixture was poured over a saturated aqueous solution of sodium bicarbonate $(15 \mathrm{~mL})$ and ice, and was then extracted with dichloromethane $(3 \times 10 \mathrm{~mL})$. The organic layers were combined, dried over anhydrous magnesium sulfate, filtered, and concentrated under vacuum. Purification by column chromatography (dichloromethane) gave a white solid. Yield: $86 \mathrm{mg}, 64 \% .{ }^{1} \mathrm{H} \mathrm{NMR} \mathrm{(300} \mathrm{MHz,}$ $\left.\mathrm{CDCl}_{3}\right): \delta 7.47(\mathrm{~d}, 2 \mathrm{H}, J=3.9 \mathrm{~Hz}), 7.31(\mathrm{dd}, 2 \mathrm{H}, J=1.3,5.1 \mathrm{~Hz}), 7.24,(\mathrm{dd}, 2 \mathrm{H}, J=1.4,3.7 \mathrm{~Hz}), 7.15$ $(\mathrm{d}, 2 \mathrm{H}, J=3.9 \mathrm{~Hz}), 7.04(\mathrm{dd}, 2 \mathrm{H}, J=3.7,5.0 \mathrm{~Hz})$. HRMS (ESI): $m / z$ calculated: 378.9413 , found 378.9416. IR (neat): $(\sigma \mathrm{SO}) 1049 \mathrm{~cm}^{-1}$.

\section{Synthesis of $1 \mathrm{SO}_{2}$}

To a solution of $1(202 \mathrm{mg}, 0.558 \mathrm{mmol})$ in dichloromethane $(9.3 \mathrm{~mL})$ at $0{ }^{\circ} \mathrm{C}$ was added metachloroperoxybenzoic acid $(70 \%, 274 \mathrm{mg}, 1.11 \mathrm{mmol})$ and the mixture was stirred for $15 \mathrm{~min}$. The reaction mixture was poured over a saturated aqueous solution of sodium bicarbonate $(20 \mathrm{~mL})$ and ice and extracted with dichloromethane $(3 \times 15 \mathrm{~mL})$. The organic layers were combined, dried over anhydrous magnesium sulfate, filtered, and concentrated under vacuum. The crude material was purified 
by column chromatography (2:1 hexanes: dichloromethane) to afford a white solid. Yield: $122 \mathrm{mg}, 55 \%$. ${ }^{1} \mathrm{H}$ NMR $\left(400 \mathrm{MHz}, \mathrm{CD}_{2} \mathrm{Cl}_{2}\right): \delta 7.64(\mathrm{~d}, 2 \mathrm{H}, J=3.9 \mathrm{~Hz}), 7.34(\mathrm{dd}, 2 \mathrm{H}, J=1.2,5.1 \mathrm{~Hz}), 7.27-7.26(\mathrm{~m}$, $2 \mathrm{H}), 7.12(\mathrm{~d}, 2 \mathrm{H}, J=4.1 \mathrm{~Hz}), 7.06(\mathrm{dd}, J=3.7,5.0 \mathrm{~Hz})$. HRMS (ESI): $m / z$ calculated: 393.9284 , found 393.9285. IR (neat): $\left(\sigma \mathrm{SO}_{2}\right) 1147 \mathrm{~cm}^{-1}$, and $1328 \mathrm{~cm}^{-1}$.

\section{Synthesis of 2S}

Prepared in the same manner as $1 S$.

Yield yellow solid: 53\%. ${ }^{1} \mathrm{H}$ NMR (400 MHz, $\left.\mathrm{CD}_{2} \mathrm{Cl}_{2}\right): \delta 7.26(\mathrm{dd}, 2 \mathrm{H}, J=0.9 \mathrm{~Hz}, 5.2 \mathrm{~Hz}), 7.20(\mathrm{dd}$, $2 \mathrm{H}, J=0.9 \mathrm{~Hz}, 3.5 \mathrm{~Hz}), 7.16(\mathrm{~d}, 2 \mathrm{H}, J=3.6 \mathrm{~Hz}), 7.09(\mathrm{~d}, 2 \mathrm{H}, J=3.6 \mathrm{~Hz}), 7.07$ (d, 2H, $J=3.9 \mathrm{~Hz}), 7.05$ $(\mathrm{d}, 2 \mathrm{H}, J=3.6 \mathrm{~Hz}), 7.04(\mathrm{~d}, 2 \mathrm{H}, J=3.6 \mathrm{~Hz}), 7.03(\mathrm{~d}, 2 \mathrm{H}, J=3.6 \mathrm{~Hz})$. HRMS (ESI): $m / z$ calculated: 525.9141 , found 525.9144 .

\section{Synthesis of $2 \mathrm{SO}$}

Prepared in the same manner as $1 S O$.

Yield yellow solid: 79\%. ${ }^{1} \mathrm{H}$ NMR (400 MHz, $\left.\mathrm{CD}_{2} \mathrm{Cl}_{2}\right): \delta 7.50(\mathrm{~d}, 2 \mathrm{H}, J=4.0 \mathrm{~Hz}$ ), 7.33 (dd, 2H, $J=1.2$, $5.2 \mathrm{~Hz}), 7.27$, (dd, 2H, $J=0.9,3.5 \mathrm{~Hz}), 7.22(\mathrm{~d}, 2 \mathrm{H}, J=3.9 \mathrm{~Hz}), 7.21(\mathrm{~d}, 2 \mathrm{H}, J=3.9 \mathrm{~Hz}), 7.17(\mathrm{~d}, 2 \mathrm{H}, J$ $=3.6 \mathrm{~Hz}), 7.09(\mathrm{~d}, 1 \mathrm{H}, J=3.7 \mathrm{~Hz}), 7.08(\mathrm{~d}, 1 \mathrm{H}, J=3.7 \mathrm{~Hz})$. HRMS (ESI): $m / z$ calculated: 542.9168, found 542.9167. IR (neat): $(\sigma \mathrm{SO}) 1047 \mathrm{~cm}^{-1}$.

\section{Synthesis of $\mathbf{2} \mathrm{SO}_{2}$}

Prepared in the same manner as $1 \mathrm{SO}_{2}$.

Yield yellow solid: $45 \% .{ }^{1} \mathrm{H}$ NMR $\left(400 \mathrm{MHz}, \mathrm{CDCl}_{3}\right): \delta 7.64(\mathrm{~d}, 2 \mathrm{H}, J=3.9 \mathrm{~Hz}), 7.31$ (dd, 2H, $J=0.9$, $5.1 \mathrm{~Hz}), 7.25,(\mathrm{~d}, 2 \mathrm{H}, J=1.4,3.4 \mathrm{~Hz}), 7.23(\mathrm{~d}, 2 \mathrm{H}, J=3.6 \mathrm{~Hz}), 7.15(\mathrm{dd}, 2 \mathrm{H}, J=1.2,4.0 \mathrm{~Hz}), 7.07$ (d, $1 \mathrm{H}, J=3.7 \mathrm{~Hz}), 7.06(\mathrm{~d}, 1 \mathrm{H}, J=3.6 \mathrm{~Hz})$. HRMS (ESI): $m / z$ calculated: 557.9039 , found 557.9047 . IR (neat): $(\sigma \mathrm{SO}) 1019,1148 \mathrm{~cm}^{-1}$, and $1330 \mathrm{~cm}^{-1}$.

\section{Synthesis of $3 S$}

Prepared in the same manner as $1 S$.

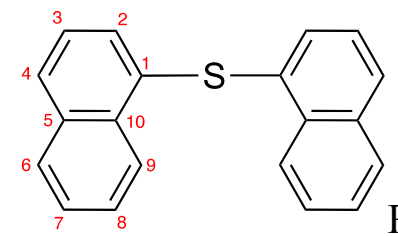

Recrystallized from DCM. Yield colorless crystals: 30\%. ${ }^{1} \mathrm{H}$ NMR $(600$ $\left.\mathrm{MHz}, \mathrm{CD}_{2} \mathrm{Cl}_{2}\right): \delta 8.45\left(\mathrm{~m}, 2 \mathrm{H}, \mathrm{H}_{9}\right), 7.95\left(\mathrm{~m}, 2 \mathrm{H}, \mathrm{H}_{6}\right), 7.85\left(\mathrm{~m}, 2 \mathrm{H}, \mathrm{H}_{4}\right), 7.58\left(\mathrm{~m}, 4 \mathrm{H}, \mathrm{H}_{7} / \mathrm{H}_{8}\right), 7.37(\mathrm{~m}$, $\left.4 \mathrm{H}, \mathrm{H}_{7} / \mathrm{H}_{8}\right) .{ }^{13} \mathrm{CNMR}\left(600 \mathrm{MHz}, \mathrm{CD}_{2} \mathrm{Cl}_{2}\right): \delta 133.69\left(\mathrm{C}_{5}\right), 132.05\left(\mathrm{C}_{10}\right), 131.85\left(\mathrm{C}_{1}\right), 129.52\left(\mathrm{C}_{2}\right), 128.13$ $\left(\mathrm{C}_{6}\right), 127.56\left(\mathrm{C}_{4}\right), 126.28\left(\mathrm{C}_{8}\right), 125.97\left(\mathrm{C}_{7}\right), 125.37\left(\mathrm{C}_{3}\right), 124.40\left(\mathrm{C}_{9}\right) .{ }^{1} \mathrm{HRMS}(\mathrm{ESI}): \mathrm{m} / z$ calculated: 286.0816 , found 286.0820 .

\section{Synthesis of $3 \mathrm{SO}$}

Prepared in the same manner as $1 S O$.

Yield white solid: $56 \%$. ${ }^{1} \mathrm{H}$ NMR (400 MHz, $\left.\mathrm{CD}_{2} \mathrm{Cl}_{2}\right): \delta 8.35(\mathrm{~m}, 2 \mathrm{H}), 8.04(\mathrm{dd}, 2 \mathrm{H}, J=0.91,7.31 \mathrm{~Hz})$, 7.99, (d, 2H, $J=7.31 \mathrm{~Hz}), 7.61(\mathrm{~d}, 1 \mathrm{H}), 7.58$ (d, 1H, $J=8.22 \mathrm{~Hz}), 7.55$ (m, 4H). HRMS (ESI): calculated $\mathrm{m} / \mathrm{z}$ 303.0844, found 303.0846. IR (neat): ( $\sigma \mathrm{SO}) 1041 \mathrm{~cm}^{-1}$. 


\section{Synthesis of $3 \mathrm{SO}_{2}$}

Prepared in the same manner as $1 \mathrm{SO}_{2}$.

Yield white solid: $17 \% .{ }^{1} \mathrm{H}$ NMR $\left(400 \mathrm{MHz}, \mathrm{CD}_{2} \mathrm{Cl}_{2}\right): \delta 8.64(\mathrm{dd}, 2 \mathrm{H}), 8.56(\mathrm{~m}, 2 \mathrm{H}), 8.15,(\mathrm{dd}, 2 \mathrm{H}, J=$ $8.6 \mathrm{~Hz}), 7.94(\mathrm{~m}, 2 \mathrm{H}, J=2.7,6.7 \mathrm{~Hz}), 7.72(\mathrm{~d}, 2 \mathrm{H}, J=7.6 \mathrm{~Hz}), 7.70$ (d, 2H, $J=8.3 \mathrm{~Hz}), 7.55(\mathrm{~m}, 2 \mathrm{H})$. HRMS (EI): calculated $\mathrm{m} / \mathrm{z}$ 318.07145, found 318.07117. IR (neat): $(\sigma \mathrm{SO}) 1119,1155 \mathrm{~cm}^{-1}$ and 1306 $\mathrm{cm}^{-1}$.

\section{Synthesis of $4 \mathrm{~S}$}

Prepared in the same manner as $1 S$.

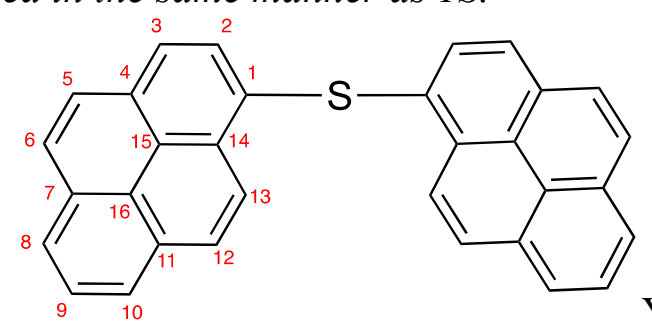

Yield yellow solid $86 \% .{ }^{1} \mathrm{H}$ NMR $\left(400 \mathrm{MHz}, \mathrm{CD}_{2} \mathrm{Cl}_{2}\right): \delta$ $8.77\left(\mathrm{~d}, 2 \mathrm{H}, J=9.14 \mathrm{~Hz}, \mathrm{H}_{13}\right), 8.25\left(\mathrm{~d}, 4 \mathrm{H}, J=7.61 \mathrm{~Hz}, \mathrm{H}_{10}\right), 8.17$, (d, $\left.2 \mathrm{H}, J=9.14 \mathrm{~Hz}, \mathrm{H}_{8}\right), 8.12$ (d, $\left.1 \mathrm{H}, J=8.83 \mathrm{~Hz}, \mathrm{H}_{12}\right), 7.08\left(\mathrm{~d}, 1 \mathrm{H}, J=7.31 \mathrm{~Hz}, \mathrm{H}_{6}\right), 8.05\left(\mathrm{~d}, 2 \mathrm{H}, J=7.31 \mathrm{~Hz}, \mathrm{H}_{9}\right), 8.04(\mathrm{~d}, 2 \mathrm{H}, J=8.83$ $\left.\mathrm{Hz}, \mathrm{H}_{5}\right), 8.03\left(\mathrm{~d}, 2 \mathrm{H}, J=8.22 \mathrm{~Hz}, \mathrm{H}_{3}\right), 7.85\left(\mathrm{~d}, 2 \mathrm{H}, J=8.22 \mathrm{~Hz}, \mathrm{H}_{2}\right) .{ }^{13} \mathrm{CNMR}\left(600 \mathrm{MHz}, \mathrm{CD}_{2} \mathrm{Cl}_{2}\right): \delta$ $131.91\left(\mathrm{C}_{7}\right), 131.57\left(\mathrm{C}_{11}\right), 131.38\left(\mathrm{C}_{4}\right), 131.30\left(\mathrm{C}_{14}\right), 130.90\left(\mathrm{C}_{1}\right), 130.58\left(\mathrm{C}_{2}\right), 128.92\left(\mathrm{C}_{12}\right), 128.25$ $\left(\mathrm{C}_{6}\right), 127.79\left(\mathrm{C}_{5}\right), 126.95\left(\mathrm{C}_{9}\right), 126.13\left(\mathrm{C}_{8}\right), 126.08\left(\mathrm{C}_{10}\right), 125.89\left(\mathrm{C}_{15}\right), 125.80\left(\mathrm{C}_{3}\right), 124.97\left(\mathrm{C}_{16}\right)$, $124.75\left(\mathrm{C}_{12}\right)$. HRMS (ESI): calculated $m / z 434.1129$, found 434.1128 .

\section{Synthesis of 4SO}

Prepared in the same manner as $1 S O$.

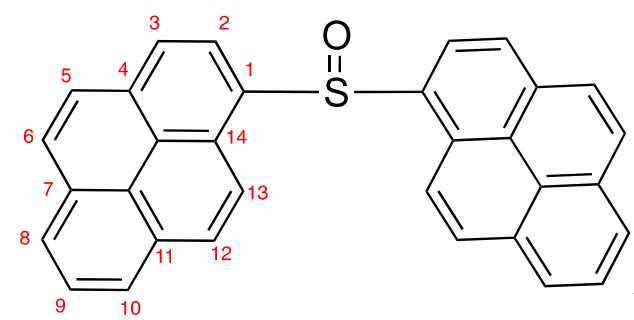

Yield yellow solid: $30 \% .{ }^{1} \mathrm{H}$ NMR (400 MHz, $\mathrm{CD}_{2} \mathrm{Cl}_{2}$ ): $\delta$ $8.75\left(\mathrm{~d}, 2 \mathrm{H}, J=9.22 \mathrm{~Hz}, \mathrm{H}_{13}\right), 8.56\left(\mathrm{~d}, 2 \mathrm{H}, J=8.19 \mathrm{~Hz}, \mathrm{H}_{2}\right), 8.29$, (d, $\left.2 \mathrm{H}, J=8.19 \mathrm{~Hz}, \mathrm{H}_{3}\right), 8.27$ (d, $\left.2 \mathrm{H}, J=8.88 \mathrm{~Hz}, \mathrm{H}_{8}\right), 8.25\left(\mathrm{~d}, 2 \mathrm{H}, J=8.19 \mathrm{~Hz}, \mathrm{H}_{10}\right), 8.20\left(\mathrm{~d}, 2 \mathrm{H}, J=9.56 \mathrm{~Hz}, \mathrm{H}_{12}\right), 8.17(\mathrm{~d}, 2 \mathrm{H}, J=$ $\left.9.22 \mathrm{~Hz}, \mathrm{H}_{6}\right), 8.09\left(\mathrm{~d}, 2 \mathrm{H}, J=8.88 \mathrm{~Hz}, \mathrm{H}_{5}\right), 8.07\left(1 \mathrm{H}, J=7.51, \mathrm{H}_{9}\right), 8.05\left(1 \mathrm{H}, J=7.85, \mathrm{H}_{9}\right) .{ }^{13} \mathrm{CNMR}$ $\left(400 \mathrm{MHz}, \mathrm{CD}_{2} \mathrm{Cl}_{2}\right): \delta 137.76\left(\mathrm{C}_{1}\right), 133.43\left(\mathrm{C}_{4}\right), 131.23\left(\mathrm{C}_{7}\right), 130.52\left(\mathrm{C}_{11}\right), 129.64\left(\mathrm{C}_{12}\right), 129.41\left(\mathrm{C}_{6}\right)$, $128.67\left(\mathrm{C}_{14}\right), 127.28\left(\mathrm{C}_{5}\right), 126.77\left(\mathrm{C}_{9}\right), 126.49\left(\mathrm{C}_{10}\right), 125.60\left(\mathrm{C}_{3}\right), 124.65\left(\mathrm{C}_{15}\right), 124.34\left(\mathrm{C}_{16}\right), 123.46$ $\left(\mathrm{C}_{2}\right), 121.50\left(\mathrm{C}_{13}\right)$ HRMS (EI): calculated $\mathrm{m} / z$ 450.10784, found 450.10760. IR (neat): $(\sigma \mathrm{SO}) 1046 \mathrm{~cm}^{-1}$

\section{Synthesis of $\mathbf{4 S O}_{2}$}

Prepared in the same manner as $1 \mathrm{SO}_{2}$.

Yield yellow solid: $24 \% .{ }^{1} \mathrm{H}$ NMR $\left(400 \mathrm{MHz}, \mathrm{CD}_{2} \mathrm{Cl}_{2}\right): \delta 9.16(\mathrm{~d}, 2 \mathrm{H}, J=8.22 \mathrm{~Hz}), 8.95(\mathrm{~d}, 2 \mathrm{H}, J=$ $9.44 \mathrm{~Hz}), 8.43,(\mathrm{~d}, 2 \mathrm{H}, J=8.53 \mathrm{~Hz}), 8.30(\mathrm{~d}, 2 \mathrm{H}, J=7.61 \mathrm{~Hz}), 8.25(\mathrm{~d}, 4 \mathrm{H}, J=8.83 \mathrm{~Hz}), 8.17(\mathrm{dd}, 4 \mathrm{H}$, $J=2.13,9.14 \mathrm{~Hz}) 8.08(\mathrm{~d}, 1 \mathrm{H}, J=7.61 \mathrm{~Hz}), 8.06(\mathrm{~d}, 1 \mathrm{H}, J=7.61 \mathrm{~Hz})$. HRMS (ESI): $m / z$ calculated: 467.1106, found 467.1104. IR (neat): $(\sigma \mathrm{SO}) 1122,1153 \mathrm{~cm}^{-1}$, and $1296 \mathrm{~cm}^{-1}$. 


\section{DFT Calculations}

All calculations were performed with the 2012.01 version of the Amsterdam Density Functional (ADF) program. ${ }^{2}$ All electrons were included in the variational treatment (i.e., no frozen-core approximation was applied). $\mathrm{C}_{1}$ (NOSYM) symmetry-unrestricted and $\mathrm{C}_{2}$ and $\mathrm{C}_{\mathrm{s}}$ symmetry-restricted geometry optimizations for $\mathbf{1 S O}_{\mathbf{n}}$ and $\mathbf{2 S O}_{\mathbf{n}}$ yielded similar results for orbital energies and compositions in each case for different conformations of the thiophene rings relative to the central $\mathrm{S}$ atom. Energies, geometries, bond orders, ${ }^{3}$ atomic charges, ${ }^{4}$ and the orbital electronic structure were calculated using the generalized gradient approximation (GGA) of density functional theory (DFT) at the BP86 level. The GGA proceeds from the local density approximation (LDA), where exchange is described by Slater's $X \alpha$ potential $^{2 \mathrm{~h}}$ and correlation is treated in the Vosko-Wilk-Nusair (VWN) parameterization, ${ }^{2^{\mathrm{i}}}$ and is augmented with nonlocal corrections to exchange due to Becke $\mathrm{e}^{\mathrm{j}, \mathrm{k}}$ and correlation due to Perdew ${ }^{2^{1}}$ added self-consistently. ${ }^{2 \mathrm{~m}}$ Relativistic effects were taken into account in all cases using the zeroth-order relativistic approximation (ZORA) $)^{5}$ and all-electron TZ2P basis sets from the ADF ZORA basis sets library were used for all atoms. ${ }^{2 f}$ TDDFT excited state geometries for $\mathbf{2 S}, \mathbf{2 S O}$, and $\mathbf{2 S O 2}$ were calculated for the lowest singlet excited state of each. All other parameters were the same as for the BP86 ground state geometry optimizations. 


\section{DFT Data}
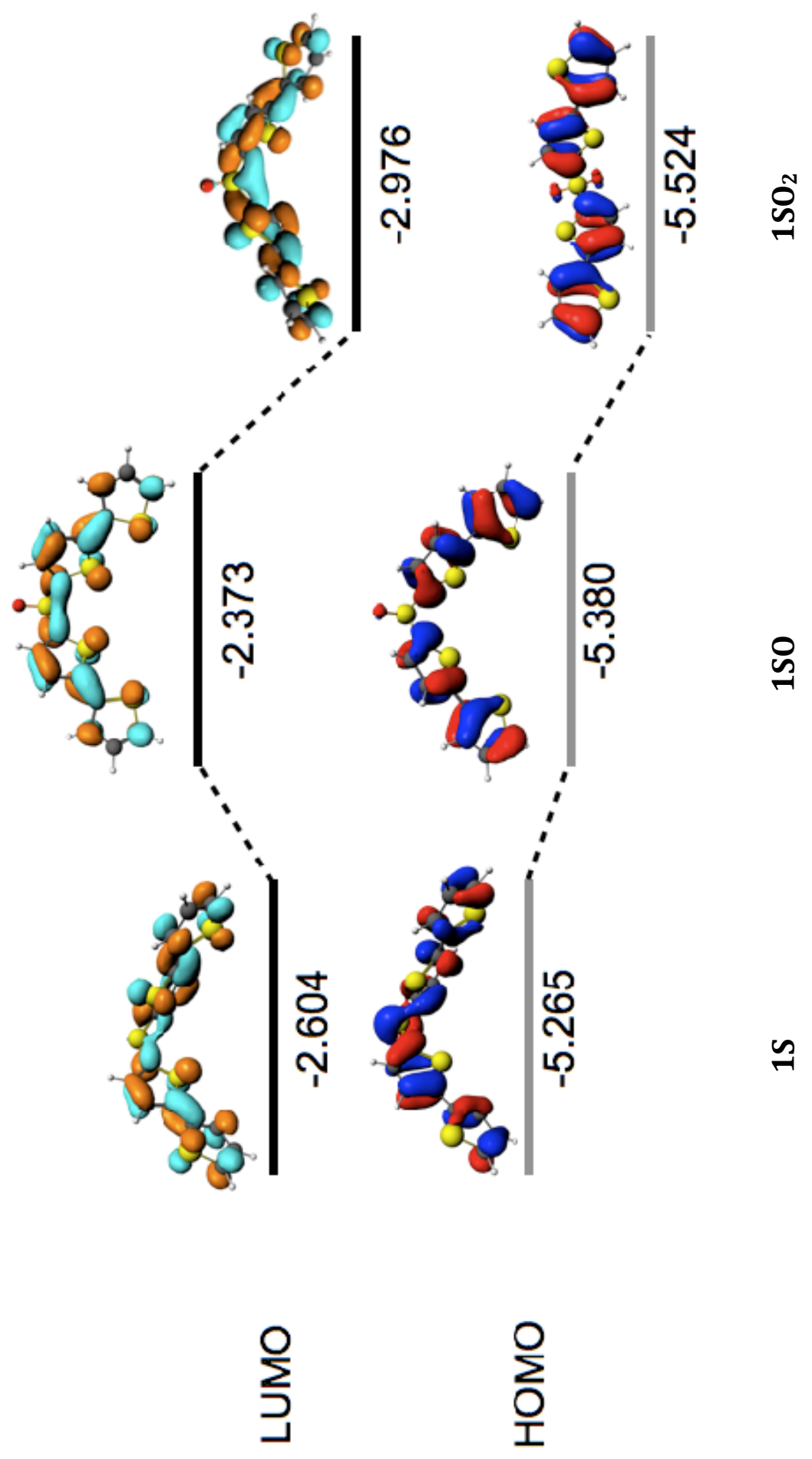

Figure S1. Calculated frontier molecular orbitals for $\mathbf{1 S O}_{\mathbf{n}}$. 


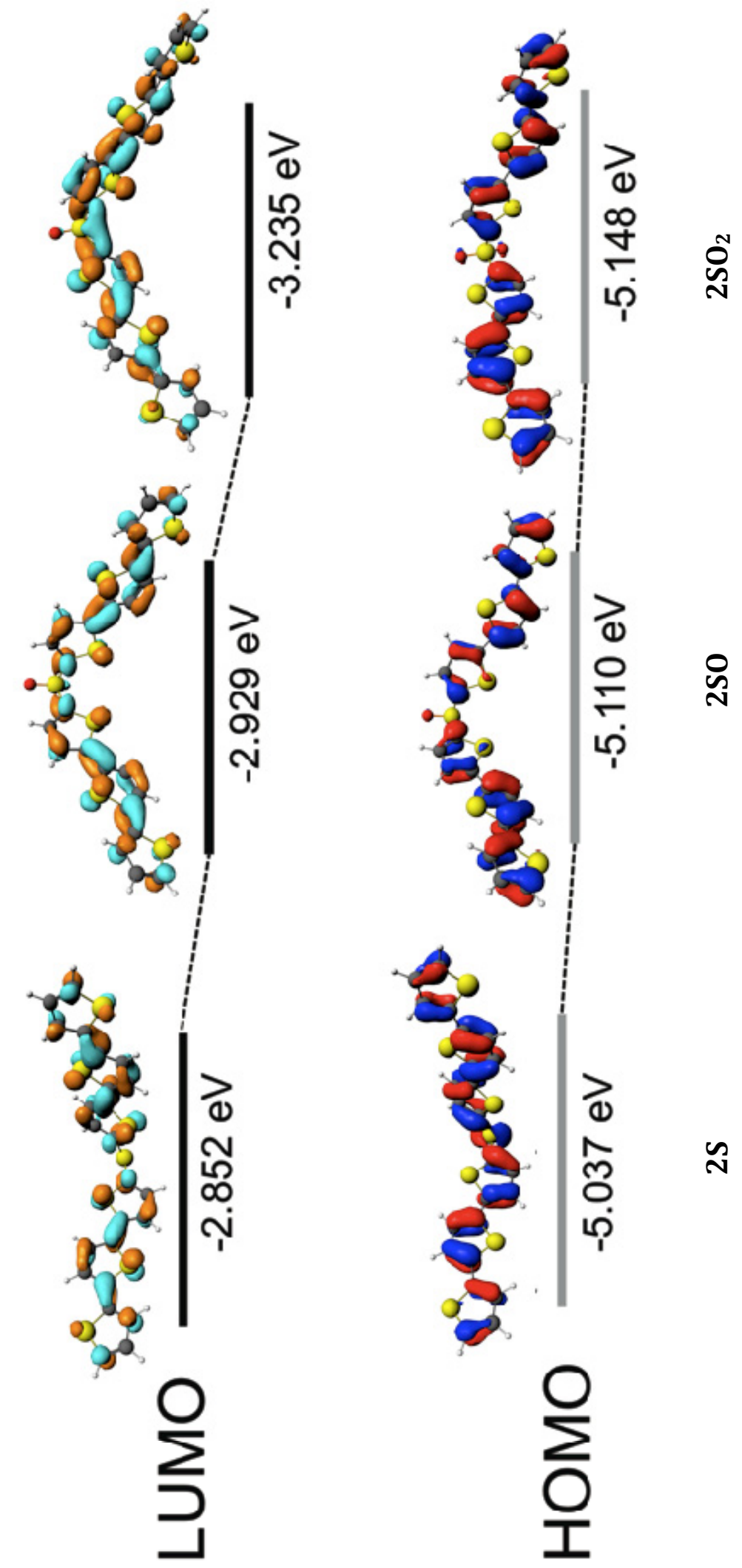

Figure S2. Calculated frontier molecular orbitals for $\mathbf{2 S O}_{\mathbf{n}}$. 
Table S1. Ground-state (GS) and excited-state (ES) atomic charges for $\mathbf{2 S O}_{\mathbf{n}}$.

$\begin{array}{lccc}\text { Compound } & \text { q Bader } & \text { q Hirshfeld } & \text { q Voronoi } \\ \text { 2S GS } & 0.174 & 0.024 & -0.001 \\ \text { 2S ES } & 0.189 & 0.053 & 0.009 \\ \text { 2S } \Delta(\mathrm{ES}-\mathrm{GS}) & 0.015 & 0.029 & 0.010 \\ & & & \\ \text { 2SO GS } & 1.351 & 0.287 & 0.314 \\ \text { 2SO ES } & 1.328 & 0.271 & 0.299 \\ \text { 2SO } \Delta(\mathrm{ES}-\mathrm{GS}) & -0.023 & -0.016 & -0.015 \\ & & & \\ \text { 2SO } & & 0.399 & 0.451 \\ \text { 2SO } & 2.550 & 0.384 & 0.441 \\ \text { 2SO } \mathrm{SO}_{2} \Delta(\mathrm{ES}-\mathrm{GS}) & -0.019 & -0.015 & -0.010\end{array}$

Table S2. Ground-state (GS) and excited-state (ES) bond lengths, and angles for $\mathbf{2 S O}_{\mathbf{n}}$.
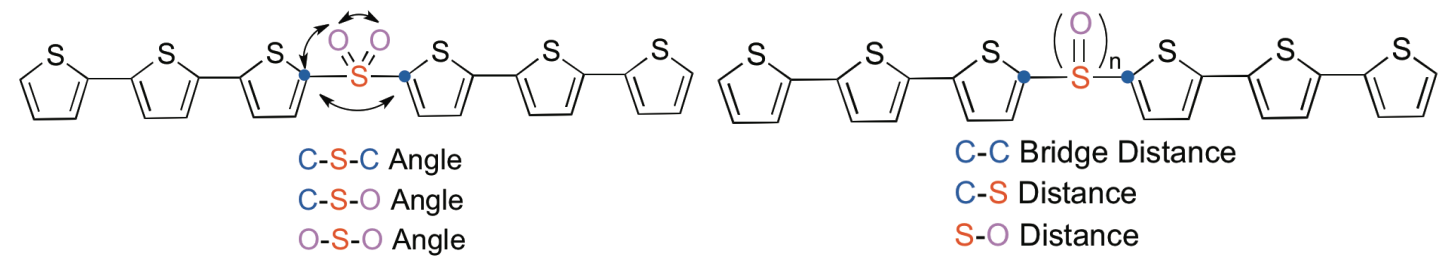

\begin{tabular}{|c|c|c|c|c|c|c|c|}
\hline Compound & $\begin{array}{l}\text { C-S-C } \\
\text { Angle }\end{array}$ & $\begin{array}{l}\text { C-S-O } \\
\text { Angle }\end{array}$ & $\begin{array}{l}\text { O-S-O } \\
\text { Angle }\end{array}$ & $\begin{array}{c}\text { C-S } \\
\text { Distance } \\
(\mathbf{p m})\end{array}$ & $\begin{array}{c}\text { S-O } \\
\text { Distance } \\
\text { (pm) }\end{array}$ & $\begin{array}{c}\text { C-C } \\
\text { bridge } \\
\text { center }\end{array}$ & $\begin{array}{c}\text { C-C } \\
\text { bridge } \\
\text { outer }\end{array}$ \\
\hline 2S GS & 105.0 & & & 175.8 & & 144.0 & 144.1 \\
\hline 2S ES & 105.8 & & & 175.0 & & 141.3 & 142.0 \\
\hline $2 \mathrm{~S} \Delta(\mathrm{ES}-\mathrm{GS})$ & 0.8 & & & -0.8 & & -2.7 & -1.9 \\
\hline 2SO GS & 101.4 & 105.2 & & 180.3 & 176.3 & 144.3 & 144.3 \\
\hline 2SO ES & 101.3 & 105.7 & & 179.6 & 175.5 & 141.5 & 141.8 \\
\hline $2 \mathrm{SO} \Delta(\mathrm{ES}-\mathrm{GS})$ & 0.0 & 0.5 & & -0.7 & -0.8 & -2.8 & -2.5 \\
\hline $2 \mathrm{SO}_{2} \mathrm{GS}$ & 108.0 & 106.3 & 123.2 & 176.3 & 145.5 & 144.3 & 144.2 \\
\hline $2 \mathrm{SO}_{2} \mathrm{ES}$ & 109.8 & 106.0 & 123.5 & 175.5 & 145.7 & 142.1 & 141.8 \\
\hline $2 \mathrm{SO}_{2} \Delta(\mathrm{ES}-\mathrm{GS})$ & 1.8 & -0.3 & 0.2 & -0.8 & 0.2 & -2.2 & -2.4 \\
\hline
\end{tabular}




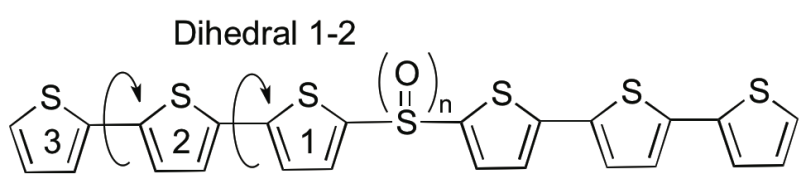

Dihedral 2-3

Table S3. Calculated thiophene dihedral angles for $\mathbf{2 S O}_{\mathbf{n}}$.

$\begin{array}{lcc}\quad \text { Compound } & \text { Dihedral Rings 1-2 (deg) } & \text { Dihedral Rings 2-3 (deg) } \\ 2 \mathrm{~S} \mathrm{GS} & 11 & 15 \\ 2 \mathrm{~S} \mathrm{ES} & 2 & 1 \\ 2 \mathrm{~S} \Delta(\mathrm{GS}-\mathrm{ES}) & 9 & 14 \\ & & \\ 2 \mathrm{SO} G S & 24 & 6 \\ 2 \mathrm{SO} \text { ES } & 1 & 0 \\ 2 \mathrm{SO} \Delta(\mathrm{GS}-\mathrm{ES}) & 23 & 6 \\ & 11 & 7 \\ 2 \mathrm{SO}_{2} \mathrm{GS} & 3 & 2 \\ 2 \mathrm{SO}_{2} \mathrm{ES} & 8 & 5 \\ 2 \mathrm{SO}_{2} \Delta(\mathrm{GS}-\mathrm{ES}) & & \end{array}$

Table S4. Calculated ground-state (GS) and excited-state (ES) bridging-sulfur (S) total bond orders (TBO) and their differences $\left[\Delta(\mathrm{ES}-\mathrm{GS}]\right.$ for $\mathbf{2 S O}_{\mathbf{n}}$. The total bond order contains contributions between $\mathrm{S}$ and all other atoms in the molecule, largely the directly bonded $\mathrm{C}$ atoms and $\mathrm{O}$ in $\mathbf{2} \mathbf{S O}$ and $\mathbf{2 S O}_{2}$.

\begin{tabular}{lccccc}
\hline Compound & $\begin{array}{c}\text { TBO S-C } \\
\text { Mayer }\end{array}$ & $\begin{array}{c}\text { TBO S-C } \\
\text { GJ }\end{array}$ & $\begin{array}{c}\text { TBO S-C } \\
\text { NM1 }\end{array}$ & $\begin{array}{c}\text { TBO S-C } \\
\text { NM2 }\end{array}$ & $\begin{array}{c}\text { TBO S-C } \\
\text { NM3 }\end{array}$ \\
$2 \mathrm{~S} \mathrm{GS}$ & 2.3549 & 2.4404 & 2.7955 & 2.7925 & 2.7657 \\
$2 \mathrm{~S} \mathrm{ES}$ & 2.4067 & 2.4904 & 2.8292 & 2.8302 & 2.7896 \\
$2 \mathrm{~S} \Delta$ (ES - GS) & 0.0518 & 0.0500 & 0.0337 & 0.0377 & 0.0239 \\
& & & & & \\
$2 \mathrm{SO}$ GS & 3.6671 & 3.3261 & 3.8114 & 4.0686 & 3.6786 \\
$2 \mathrm{SO}$ ES & 3.6384 & 3.3058 & 3.8092 & 4.0633 & 3.6584 \\
$2 \mathrm{SO} \Delta(\mathrm{ES}-\mathrm{GS})$ & -0.0287 & -0.0203 & -0.0022 & -0.0053 & -0.0202 \\
& & & & & \\
$2 \mathrm{SO}_{2} \mathrm{GS}$ & 5.6543 & 4.4314 & 4.9053 & 6.0552 & 4.6458 \\
$2 \mathrm{SO}_{2} \mathrm{ES}$ & 5.6599 & 4.4284 & 4.9143 & 6.0545 & 4.6375 \\
$2 \mathrm{SO}_{2} \Delta$ (ES - GS) & 0.0113 & 0.0029 & 0.0090 & -0.0021 & 0.0022 \\
& & & & & \\
\hline
\end{tabular}


Table S5. Calculated ground-state (GS) and excited-state (ES) bridging-sulfur $\rightarrow$ carbon (S-C) bond orders $(\mathrm{BO})$ and their differences $[\Delta(\mathrm{ES}-\mathrm{GS})]$ for $\mathbf{2 S O}_{\mathbf{n}}$.

\begin{tabular}{|c|c|c|c|c|c|}
\hline Compound & $\begin{array}{c}\text { BO S-C } \\
\text { Mayer }\end{array}$ & $\begin{array}{c}\text { BO S-C } \\
\text { GJ }\end{array}$ & $\begin{array}{c}\text { BO S-C } \\
\text { NM1 }\end{array}$ & $\begin{array}{c}\text { BO S-C } \\
\text { NM2 }\end{array}$ & $\begin{array}{c}\text { BO S-C } \\
\text { NM3 }\end{array}$ \\
\hline $2 \mathrm{~S} \mathrm{GS}$ & 1.0647 & 1.0547 & 1.2038 & 1.2391 & 1.1970 \\
\hline $2 \mathrm{~S} E S$ & 1.0759 & 1.0672 & 1.2077 & 1.2487 & 1.1972 \\
\hline $2 \mathrm{~S} \Delta(\mathrm{ES}-\mathrm{GS})$ & 0.0112 & 0.0125 & 0.0039 & 0.0096 & 0.0002 \\
\hline 2SO GS & 0.8789 & 0.8852 & 0.9272 & 1.1132 & 0.8276 \\
\hline 2SO ES & 0.8799 & 0.8856 & 0.9354 & 1.1161 & 0.8281 \\
\hline $2 \mathrm{SO} \Delta(\mathrm{ES}-\mathrm{GS})$ & 0.0010 & 0.0004 & 0.0082 & 0.0029 & 0.0005 \\
\hline $2 \mathrm{SO}_{2} \mathrm{GS}$ & 0.9377 & 0.8241 & 0.8176 & 1.2228 & 0.6916 \\
\hline $2 \mathrm{SO}_{2} \mathrm{ES}$ & 0.9678 & 0.8410 & 0.8392 & 1.2344 & 0.7049 \\
\hline $2 \mathrm{SO}_{2} \Delta(\mathrm{ES}-\mathrm{GS})$ & 0.0301 & 0.0169 & 0.0216 & 0.0116 & 0.0133 \\
\hline
\end{tabular}




\section{Solvent Analysis}

In equation $2, \varepsilon$ is the solvent dielectric constant and $n$ is the refractive index of the solvent. The solvent polarity was adjusted by using binary mixtures of cyclohexane (CHx), dichloromethane (DCM), and acetonitrile (ACN) (Table 2).

$$
\Delta f=\frac{\varepsilon-1}{2 \varepsilon+1}-\frac{n^{2}-1}{2 n^{2}+1}
$$

For binary mixtures the dielectric constant and refractive index can be calculated using equations 3 and 4 , where $f$ is the volume fraction of solvent $\mathrm{A}$ or B.

$$
\begin{aligned}
\varepsilon_{A B} & =\varepsilon_{A} f_{A}+\varepsilon_{B} f_{B} \\
n_{A B} & =n_{A} f_{A}+n_{B} f_{B}
\end{aligned}
$$

equations S3 and S4.

\begin{tabular}{cc} 
Solvent & $\Delta f$ \\
\hline $\mathrm{ACN}$ & 0.305 \\
$\mathrm{ACN}_{80} / \mathrm{DCM}_{20}$ & 0.296 \\
$\mathrm{ACN}_{50} / \mathrm{DCM}_{50}$ & 0.279 \\
$\mathrm{ACN}_{20} / \mathrm{DCM}$ & 0.253 \\
$\mathrm{DCM}$ & 0.218 \\
$\mathrm{DCM}_{80} / \mathrm{CHx}$ & 0.205 \\
$\mathrm{DCM}_{50} / \mathrm{CHx}$ & 0.172 \\
$\mathrm{DCM}_{20} / \mathrm{CHx}$ & 0.105 \\
$\mathrm{CHx}$ & 0
\end{tabular}

Table S6. Calculated $\Delta f$ values for solvent mixtures used. 

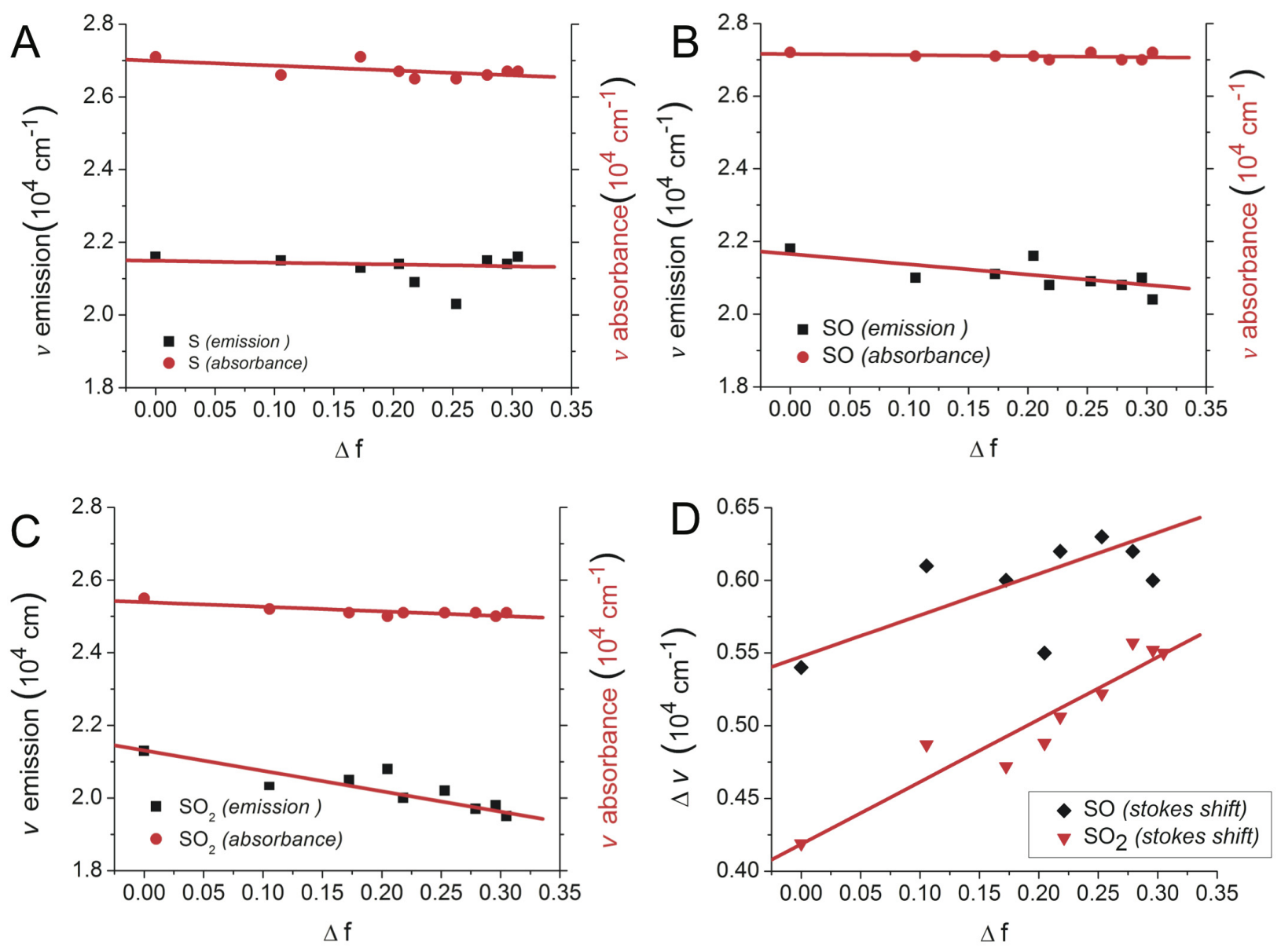

Figure S3. Lippard-Mattaga solvent-dependence analysis of series $\mathbf{2 S O}_{\mathbf{n}}$. (A-C) Change in absorbance $(\bullet)$ and emission wavenumber (-) as a function of the solvent polarity ( $\Delta f$ ). (D) Change in Stokes shift for $\mathbf{2 S O}(\checkmark), \mathbf{S O}_{2}(\boldsymbol{\nabla})$. The above data yields changes in emission energy (slope of linear fit) as (A) 0.13 ( \pm 0.07$)$, (B) $-0.30( \pm 0.10)$, and (C) $-0.50( \pm 0.10)$. Analysis of the data in (D) shows an increase in Stokes shift (slope of linear fit) for $\mathbf{2} \mathbf{S O}_{2}(0.43( \pm 0.06))$, and $\mathbf{2 S O}(0.28( \pm 0.12))$. 

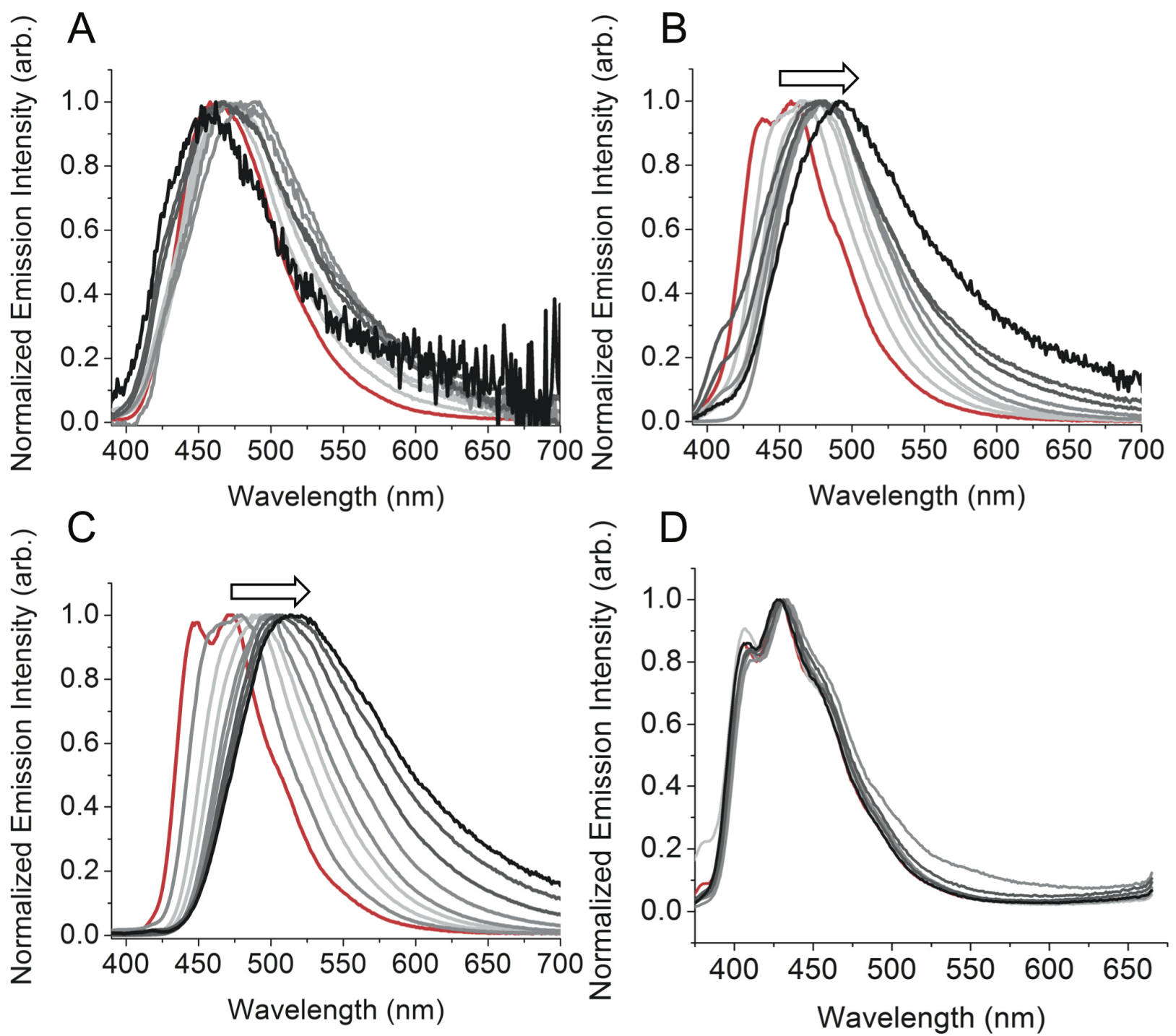

Figure S4. Normalized emission spectra for $\mathbf{2 S}$ (A), $\mathbf{2 S O}(\mathrm{B}), \mathbf{2} \mathbf{S O}_{\mathbf{2}}(\mathrm{C})$, and terthiophene (D) showing the change in emission profile in increasingly polar solvents from non-polar $\mathrm{CHx}$ (red) $\rightarrow$ ACN (black).
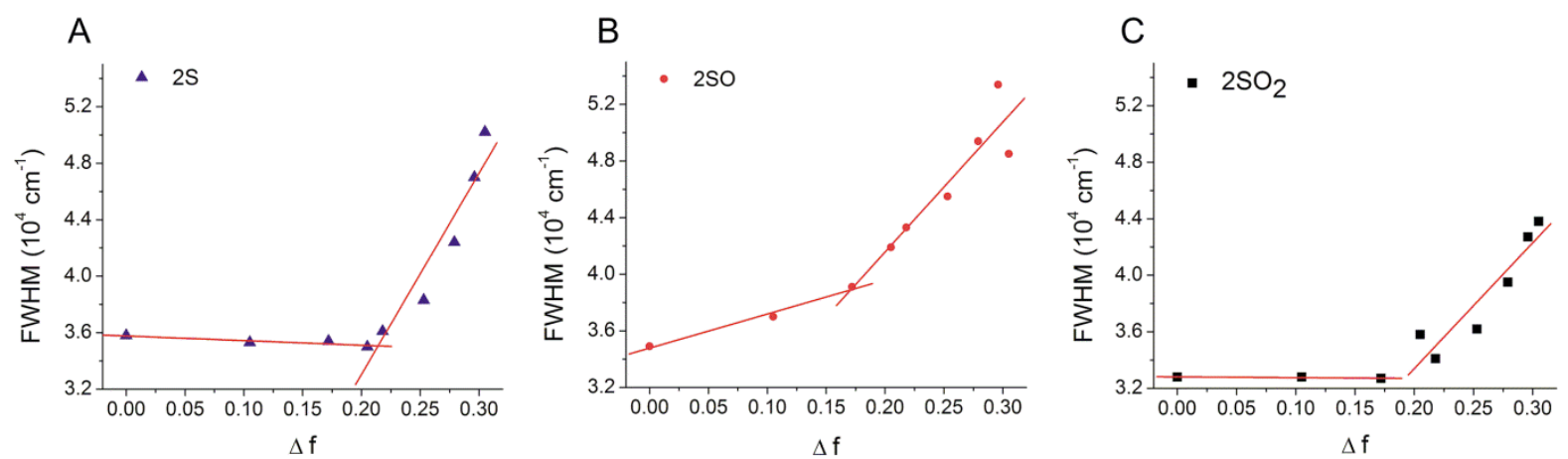

Figure S5. Full-width at half-maximum (FWHM) as a function of solvent polarity for 2S (A), 2SO (B), and $2 \mathrm{SO}_{2}(\mathrm{C})$. 


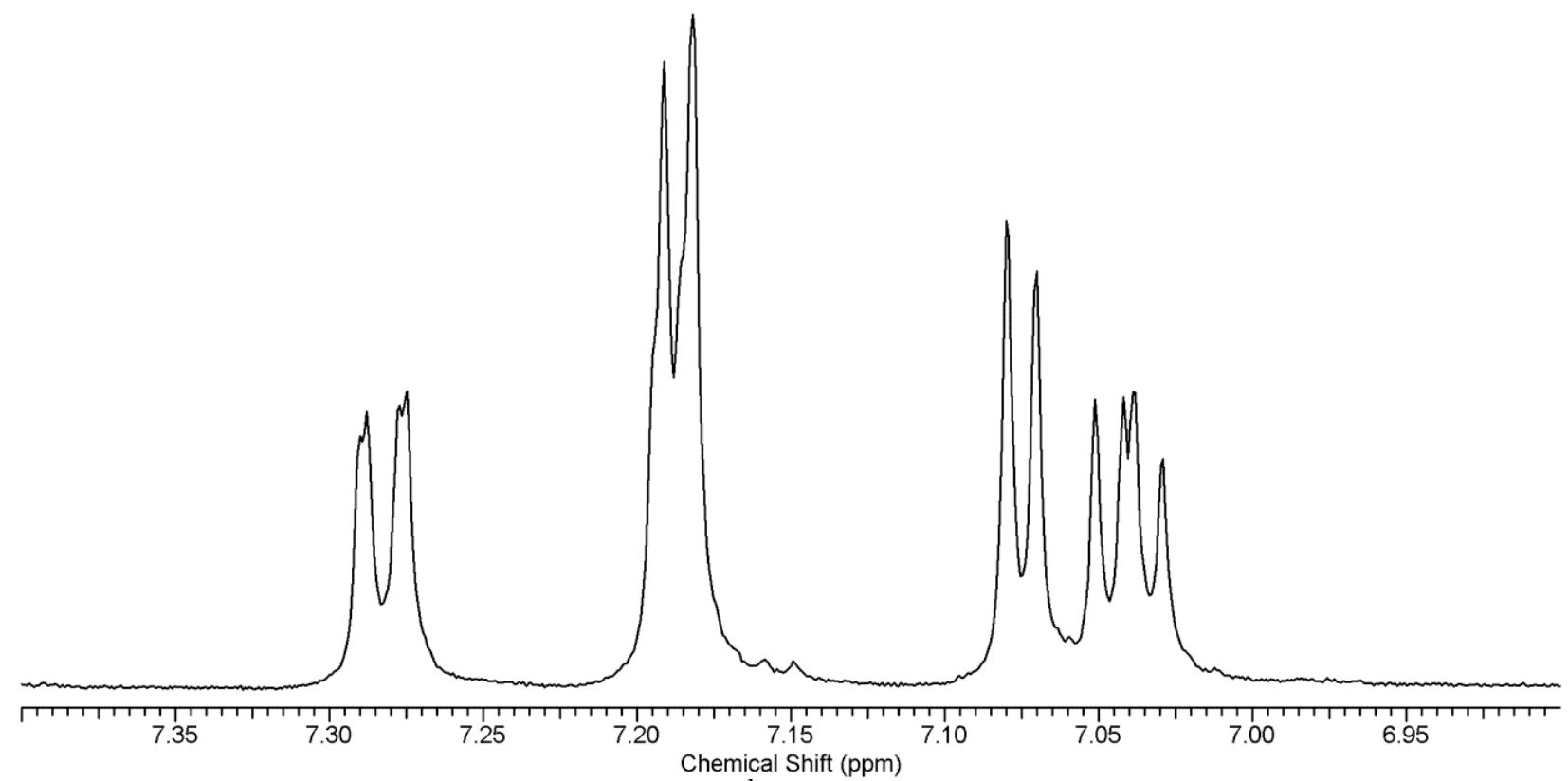

Figure S6. ${ }^{1} \mathrm{H}$ NMR spectrum of $\mathbf{1 S}$

$1 \mathrm{H}$ observe ref. to $\mathrm{CDCl} 3$ at $7.27 \mathrm{ppm}$ CHLOROFORM-d

Frequency (MHz): 300.13

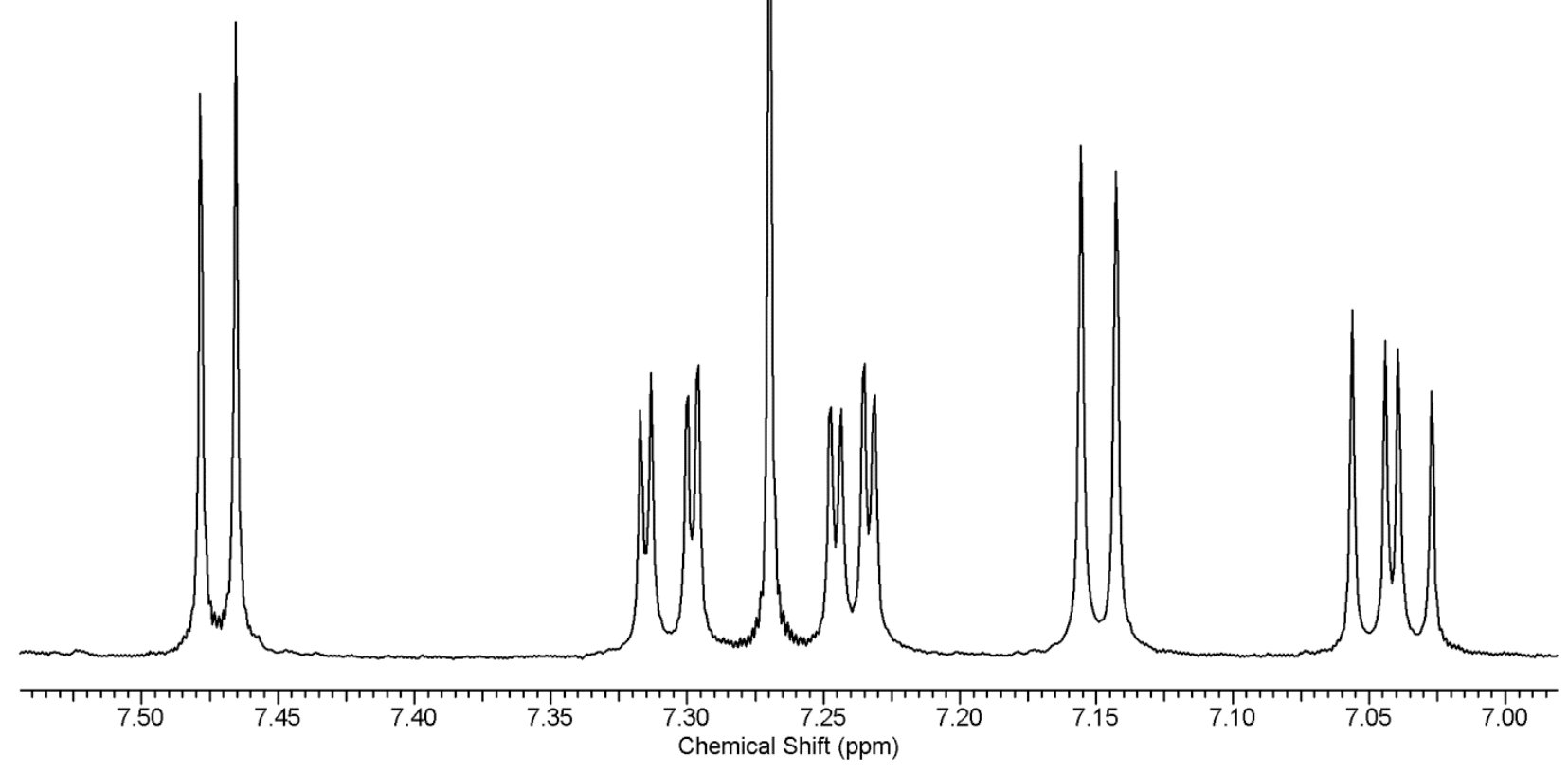


Figure S7. ${ }^{1} \mathrm{H}$ NMR spectrum of 1SO

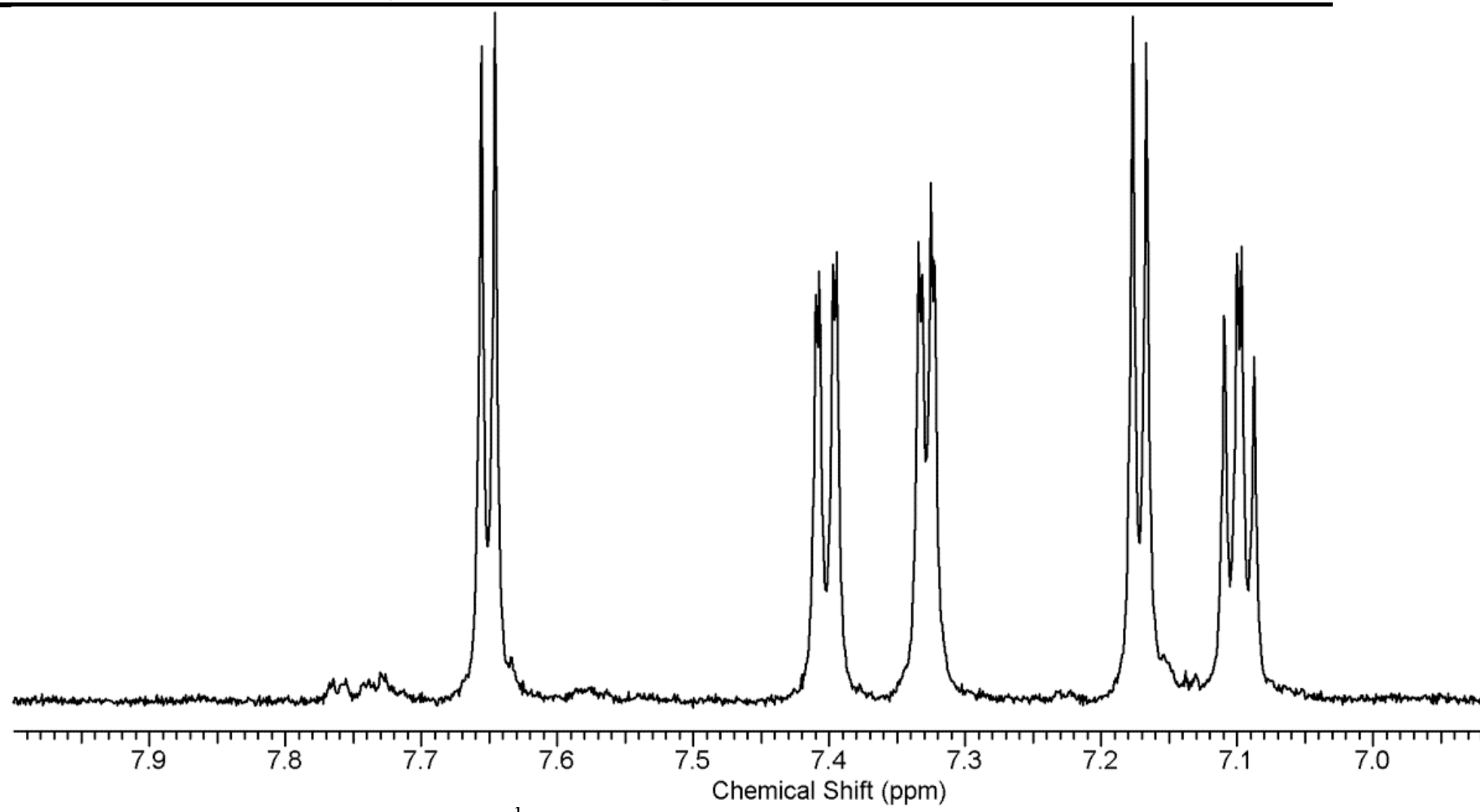

Figure S8. ${ }^{1} \mathrm{H}$ NMR spectrum of $\mathbf{1 S O}_{\mathbf{2}}$

DICHLOROMETHANE-d2

Frequency $(\mathrm{MHz}): 400.13$

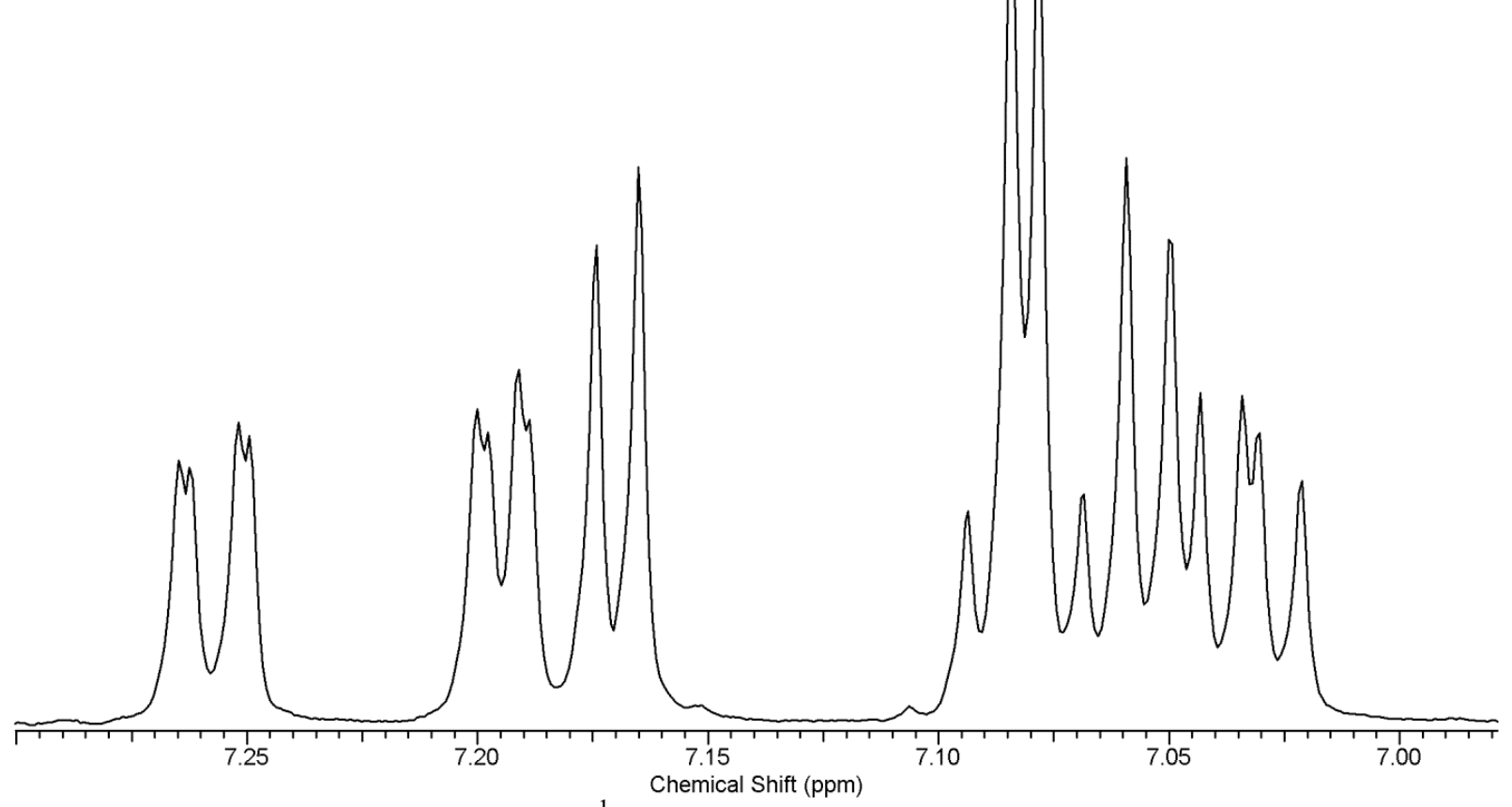

Figure S9. ${ }^{1} \mathrm{H}$ NMR spectrum of $\mathbf{2 S}$ 


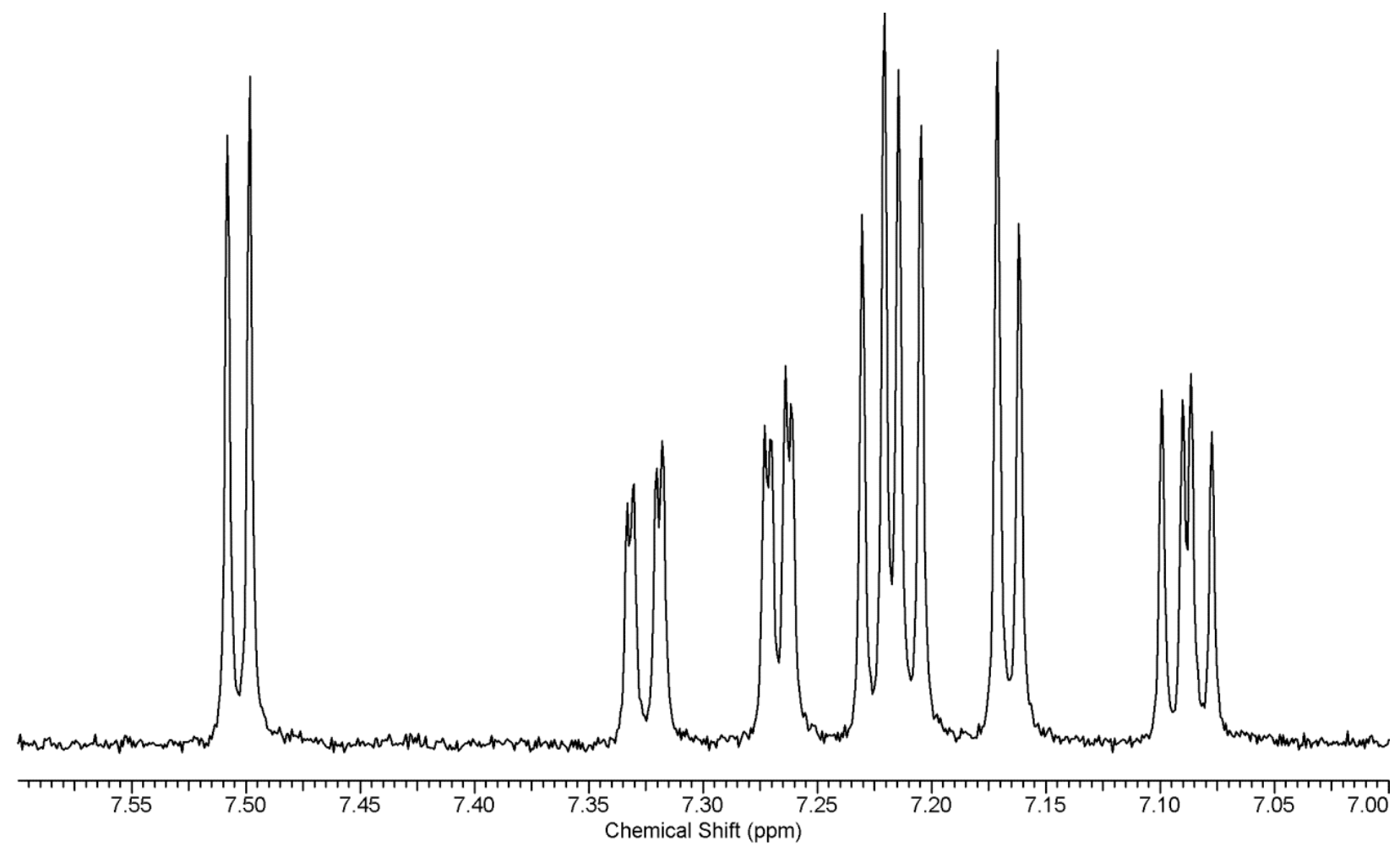

Figure S10. ${ }^{1} \mathrm{H}$ NMR spectrum of $\mathbf{2 S O}$

HRC2-U43B.UU2.esp

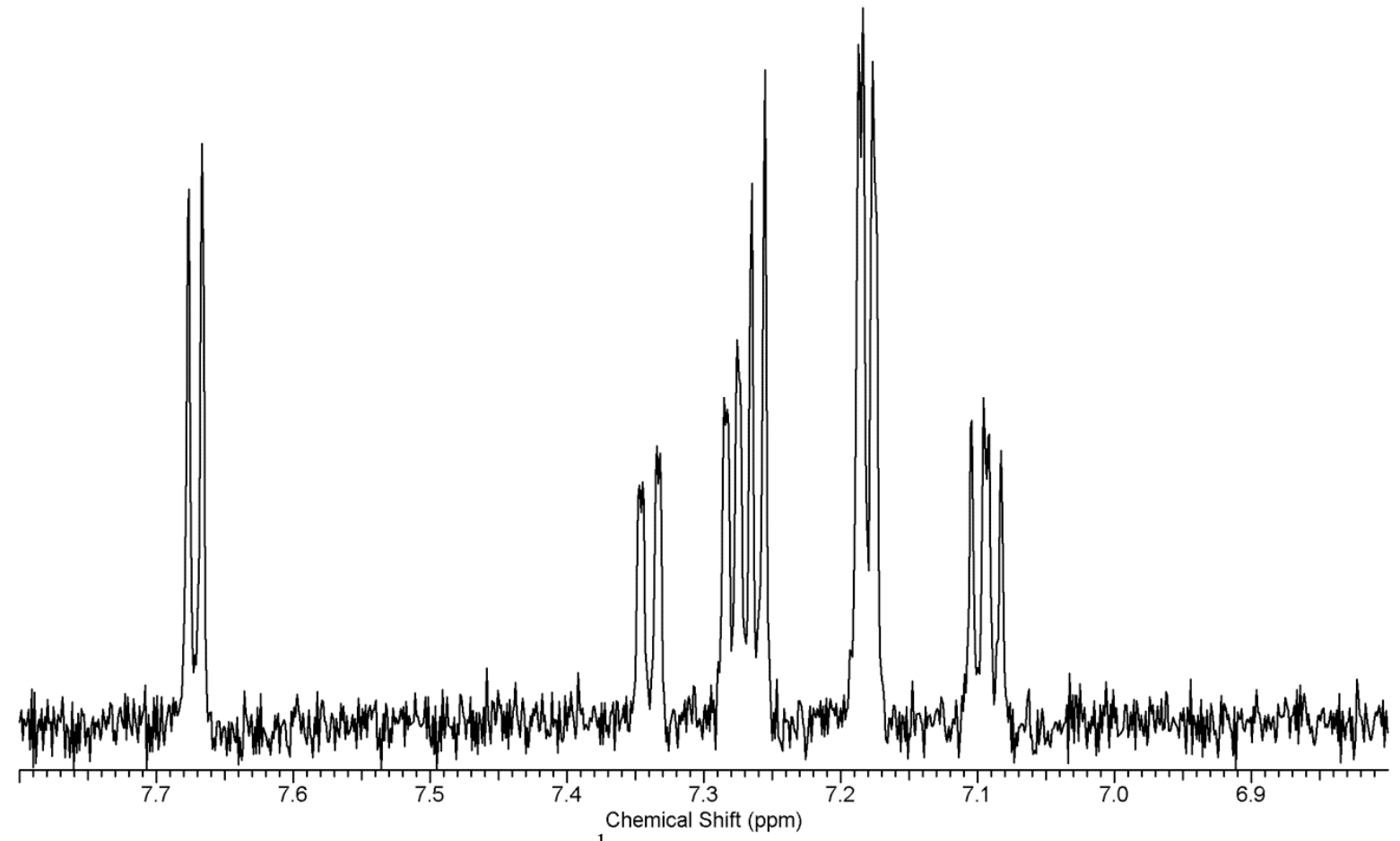

Figure S11. ${ }^{1} \mathrm{H}$ NMR spectrum of $\mathbf{2 S O}_{2}$ 
$1 \mathrm{H}$

DICHLOROMETHANE-d2

Frequency $(\mathrm{MHz}): 600.15$

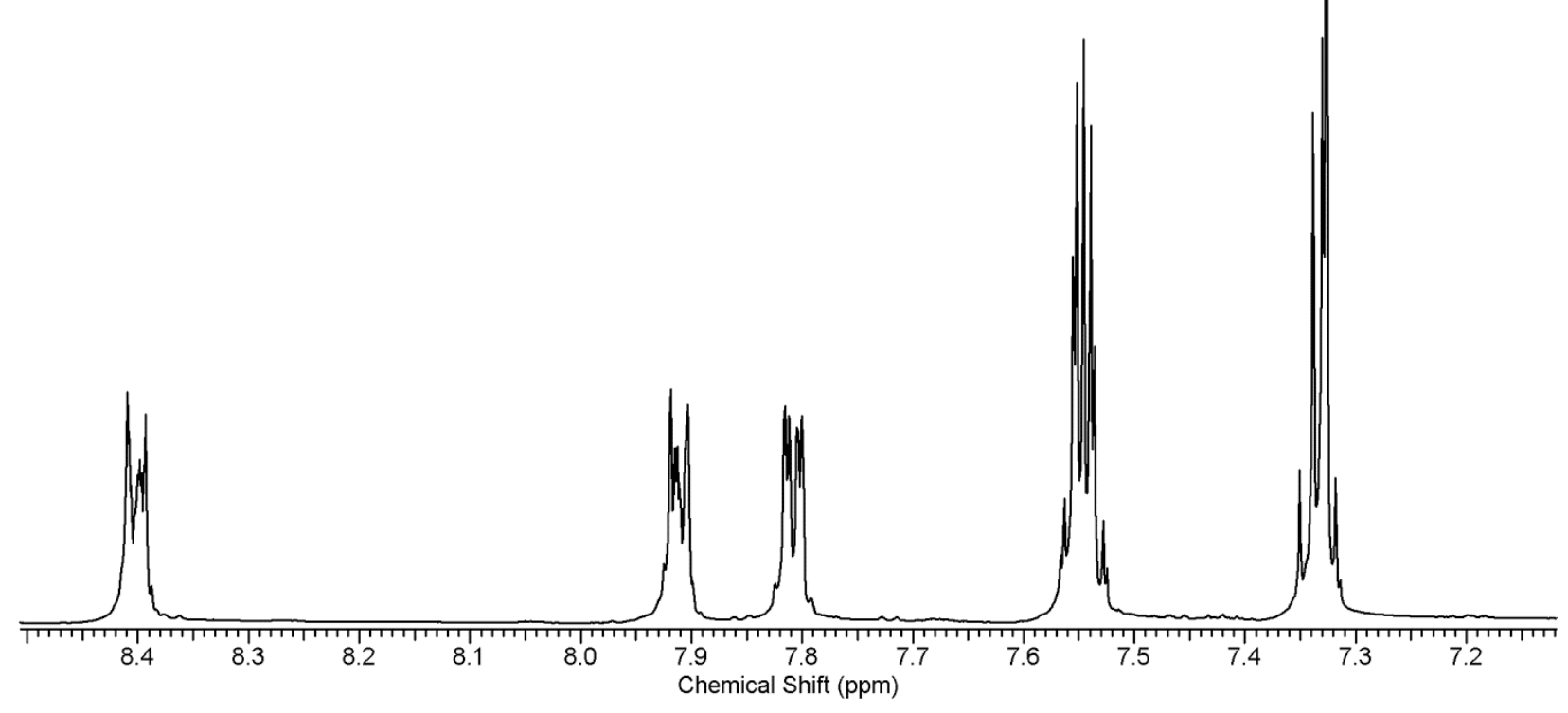

Figure S12. ${ }^{1} \mathrm{H}$ NMR spectrum of $\mathbf{3 S}$

$1 \mathrm{H}$

DICHLOROMETHANE-d2

Frequency $(\mathrm{MHz})$ : 600.15

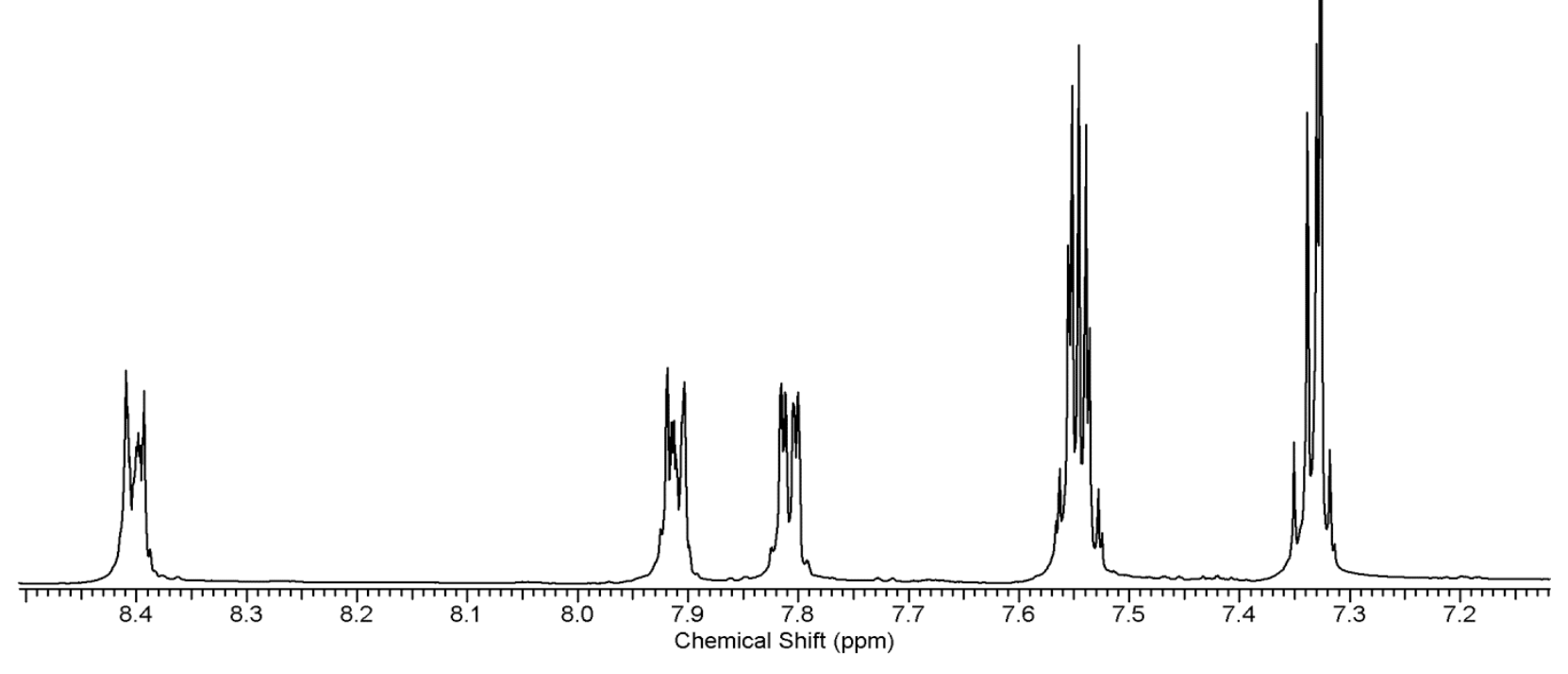

Figure S13. HSQC spectrum of $\mathbf{3 S}$ 
$5 \mathrm{~mm}$ Multinuclear inverse Z-grad Z8202/217

DICHLOROMETHANE-d2

Frequency $(\mathrm{MHz}): 400.13$

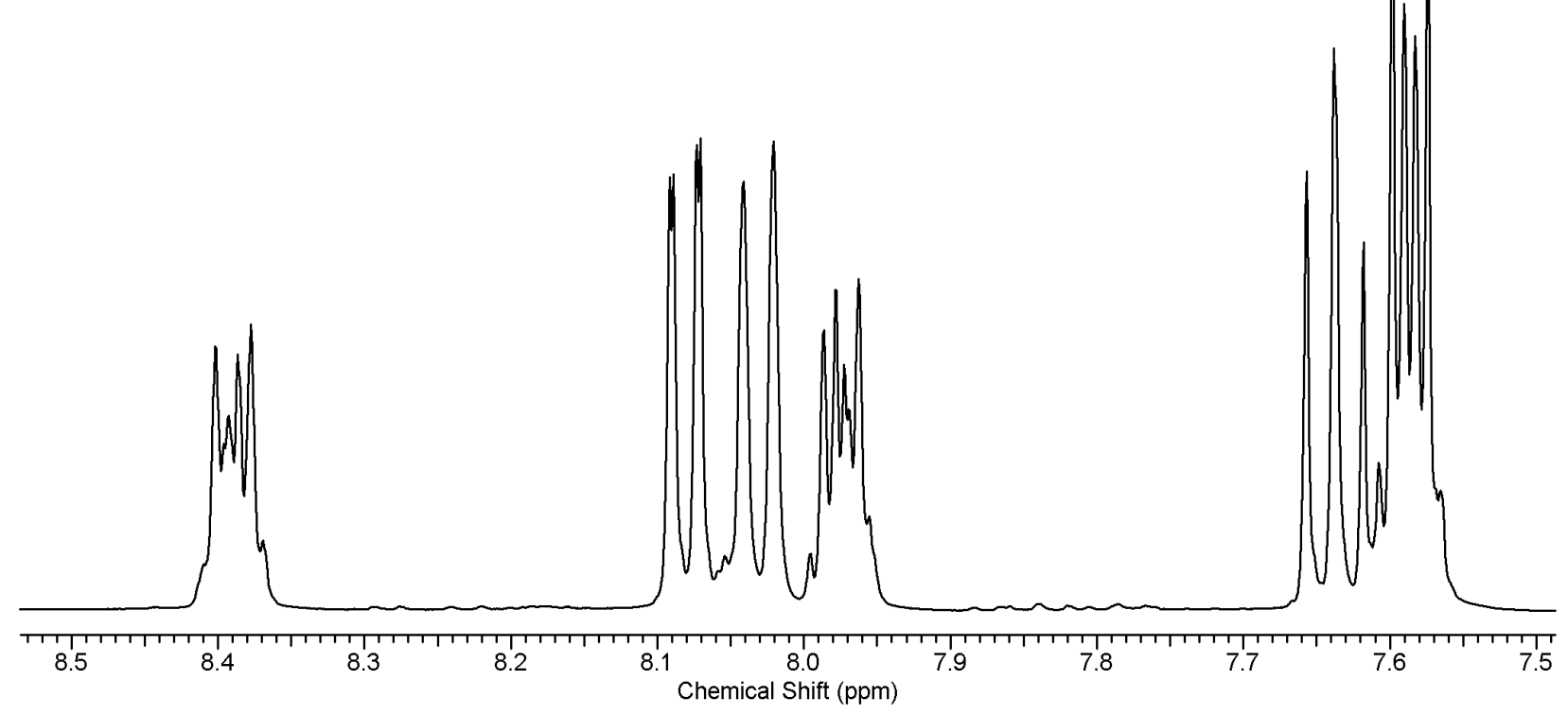

Figure S14. ${ }^{1} \mathrm{H}$ NMR spectrum of $\mathbf{3 S O}$

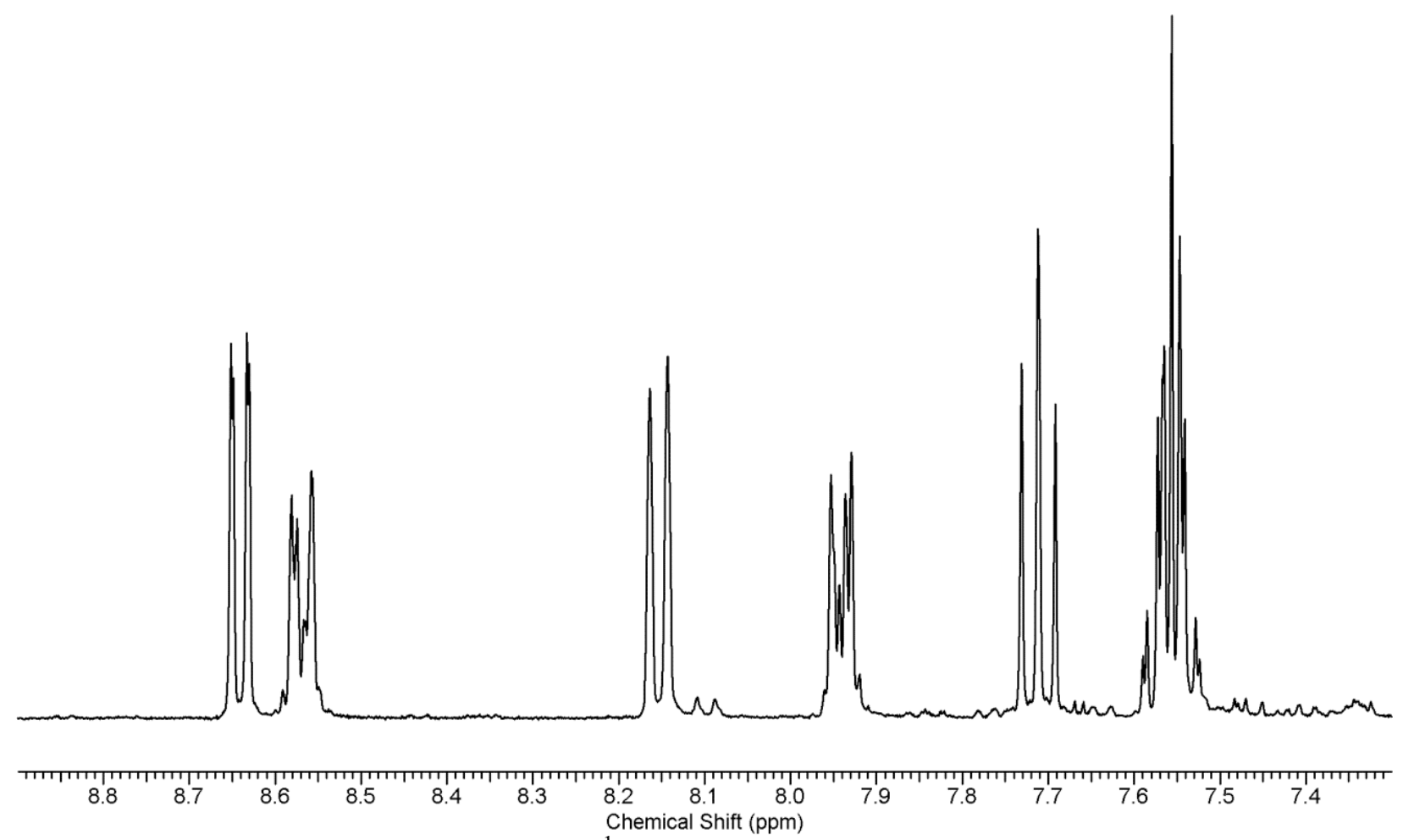

Figure S15. ${ }^{1} \mathrm{H}$ NMR spectrum of $\mathbf{3 S O}_{2}$ 


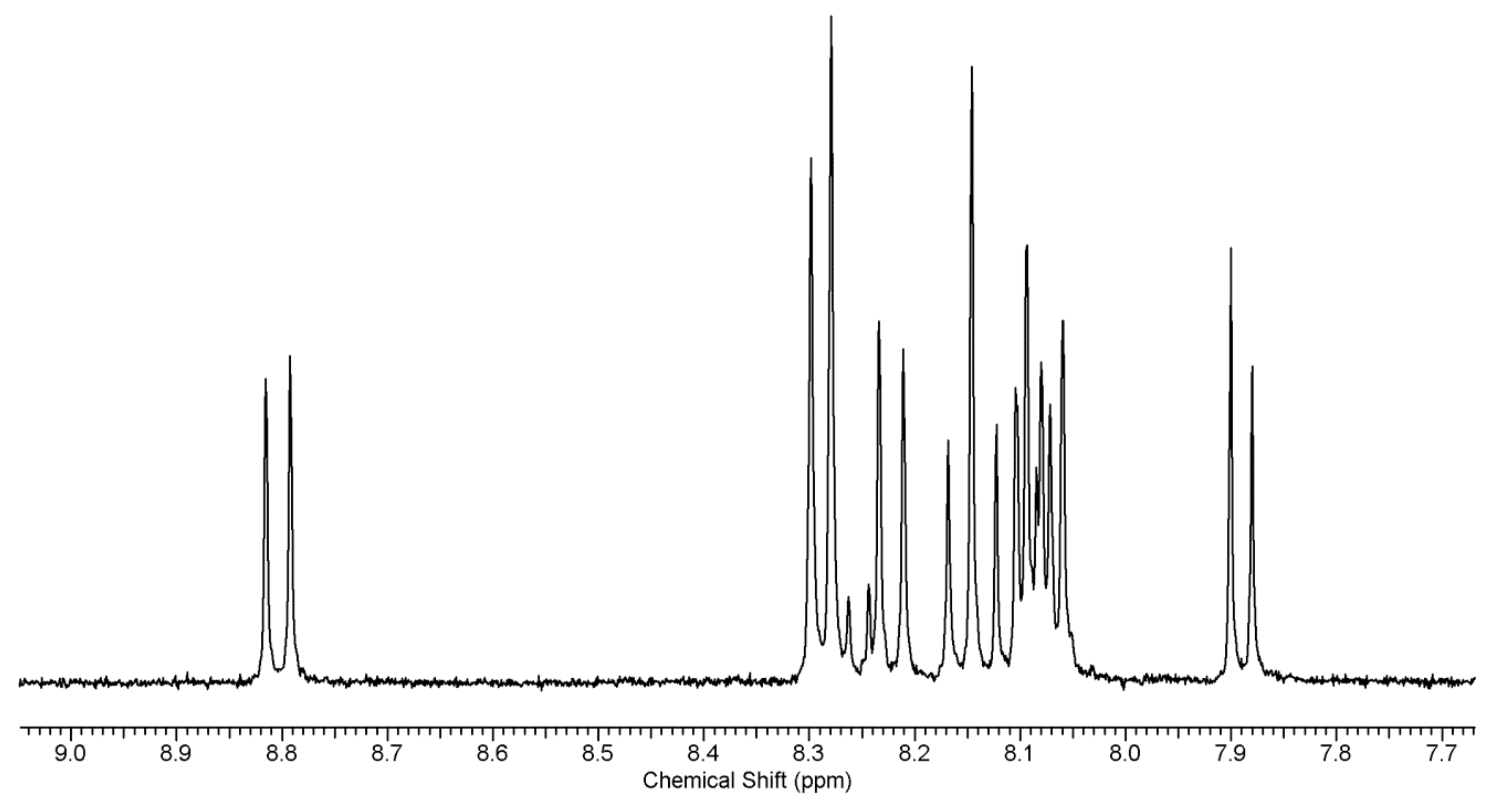

Figure S16. ${ }^{1} \mathrm{H}$ NMR spectrum of $4 \mathrm{~S}$

py-S-py.001.esp

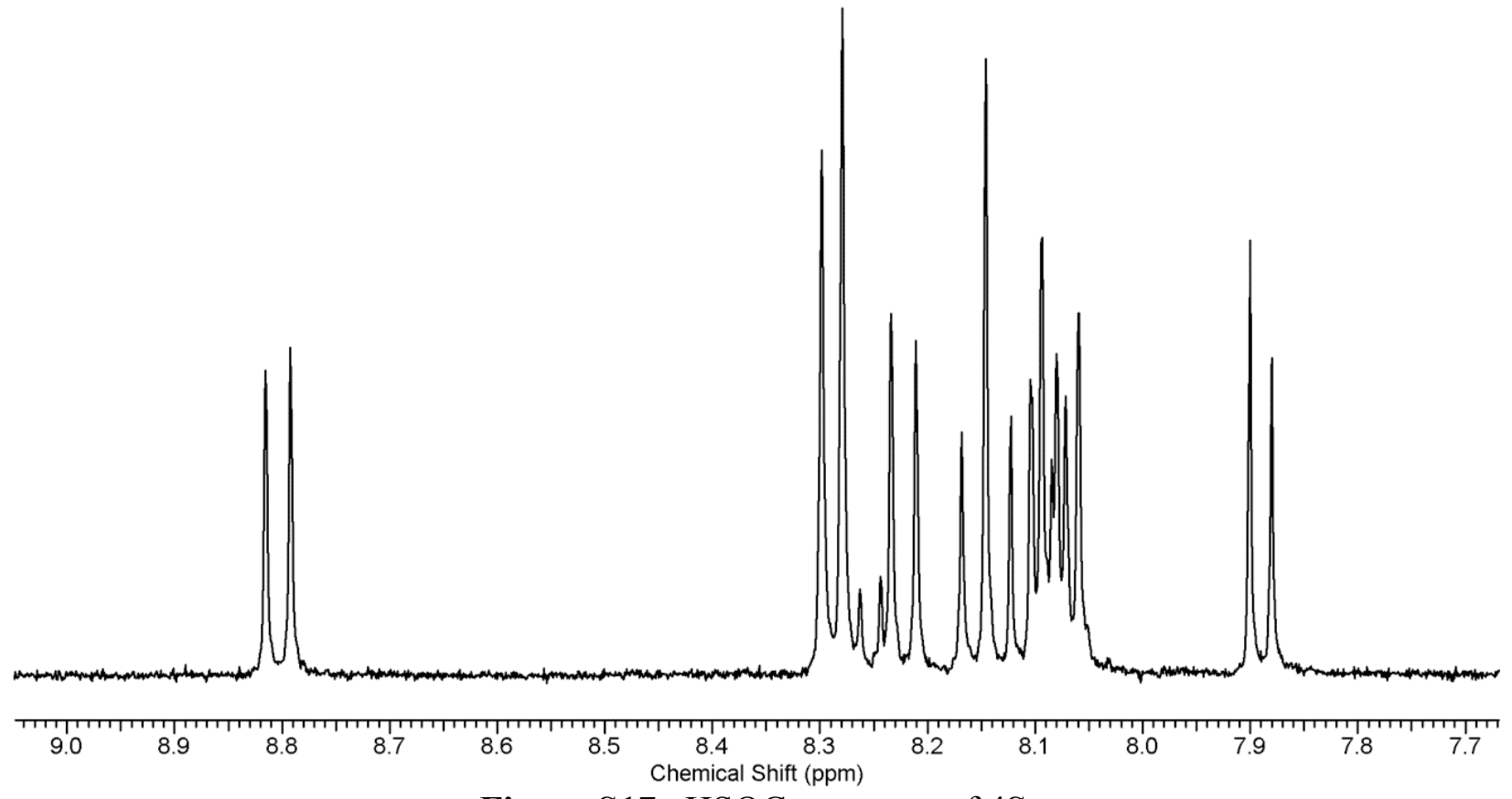

Figure S17. HSQC spectrum of $4 S$ 


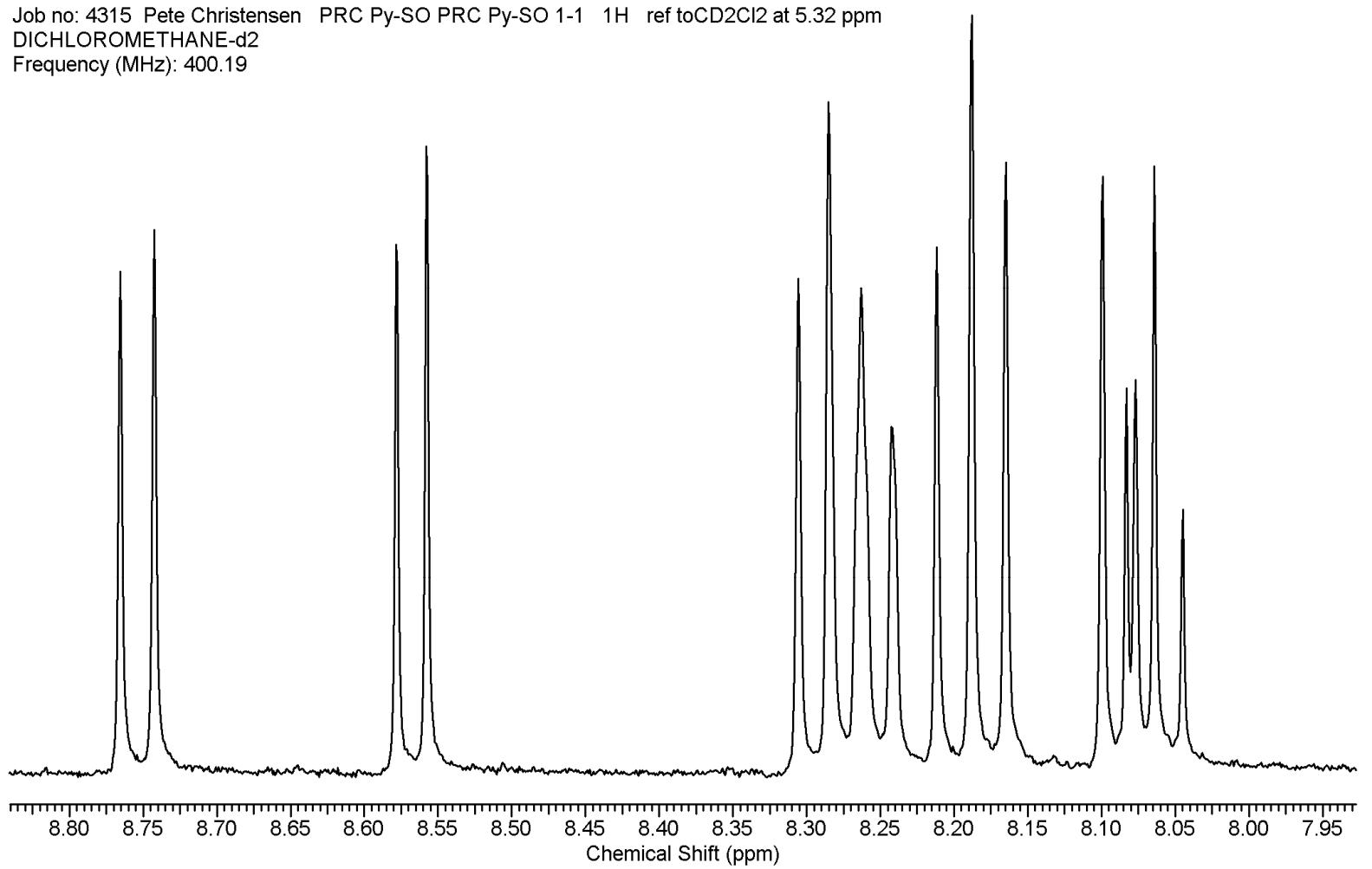

Figure S18. ${ }^{1} \mathrm{H}$ NMR spectrum of 4 SO

Job no: 4315 Pete Christensen PRC Py-SO PRC Py-SO 1-1 1H ref toCD2Cl2 at $5.32 \mathrm{ppm}$ DICHLOROMETHANE-d2 Frequency (MHz): 400.19

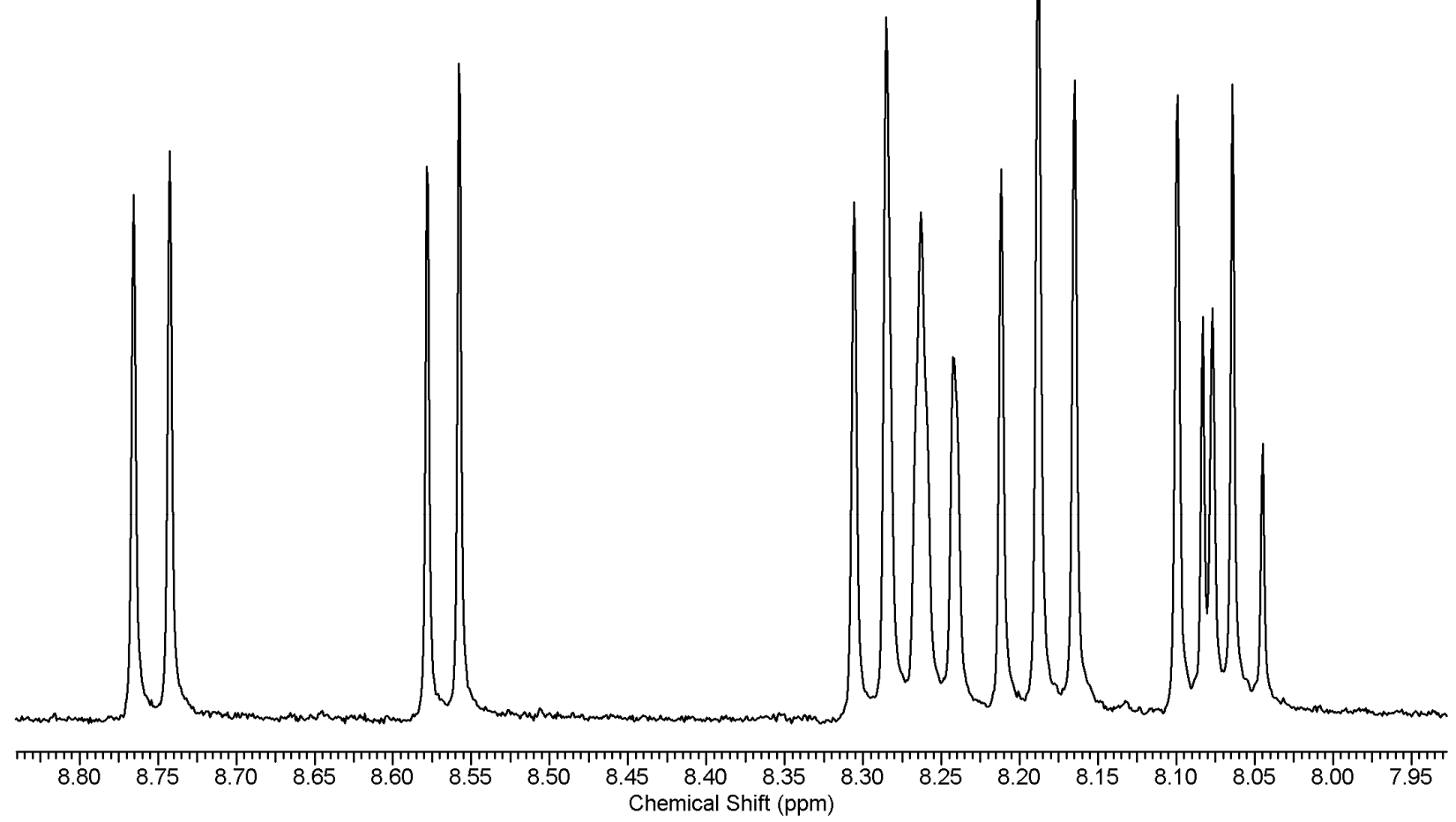

Figure S19. HSQC spectrum of 4SO 


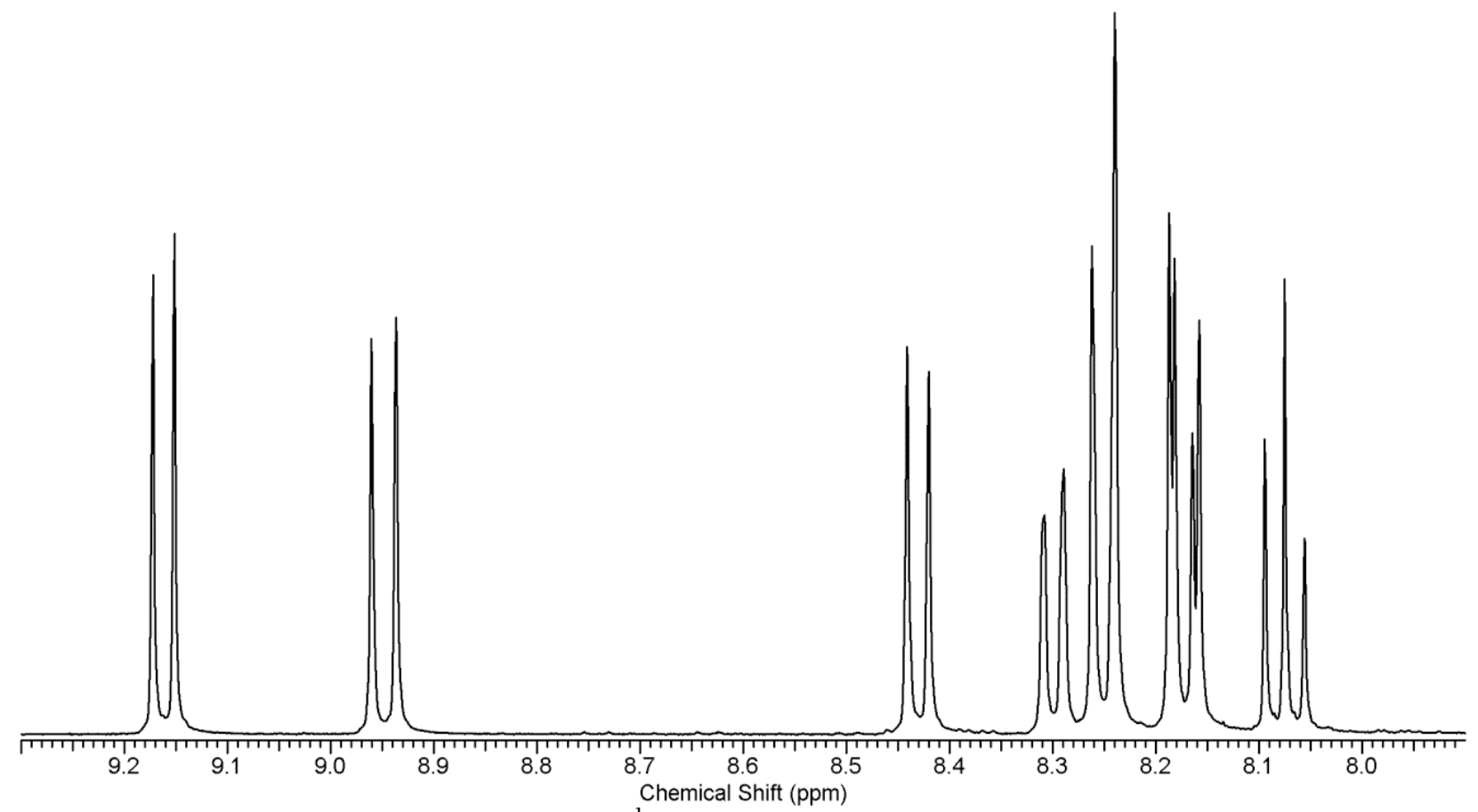

Figure S20. ${ }^{1} \mathrm{H}$ NMR spectrum of $\mathbf{4 S O}_{\mathbf{2}}$ 


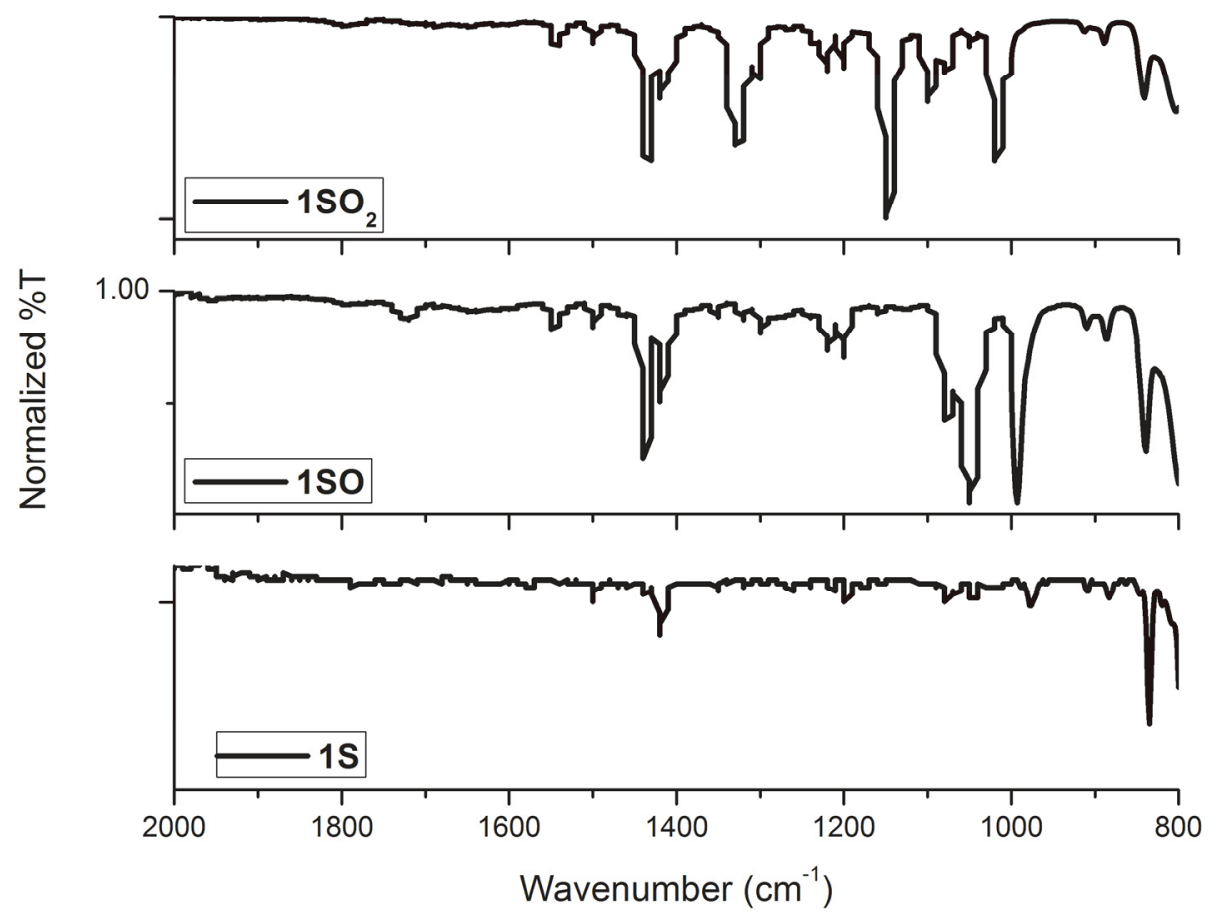

Figure S21. Infrared spectra of $\mathbf{1 S O}_{2}, \mathbf{1 S O}$ and $1 \mathrm{~S}$.

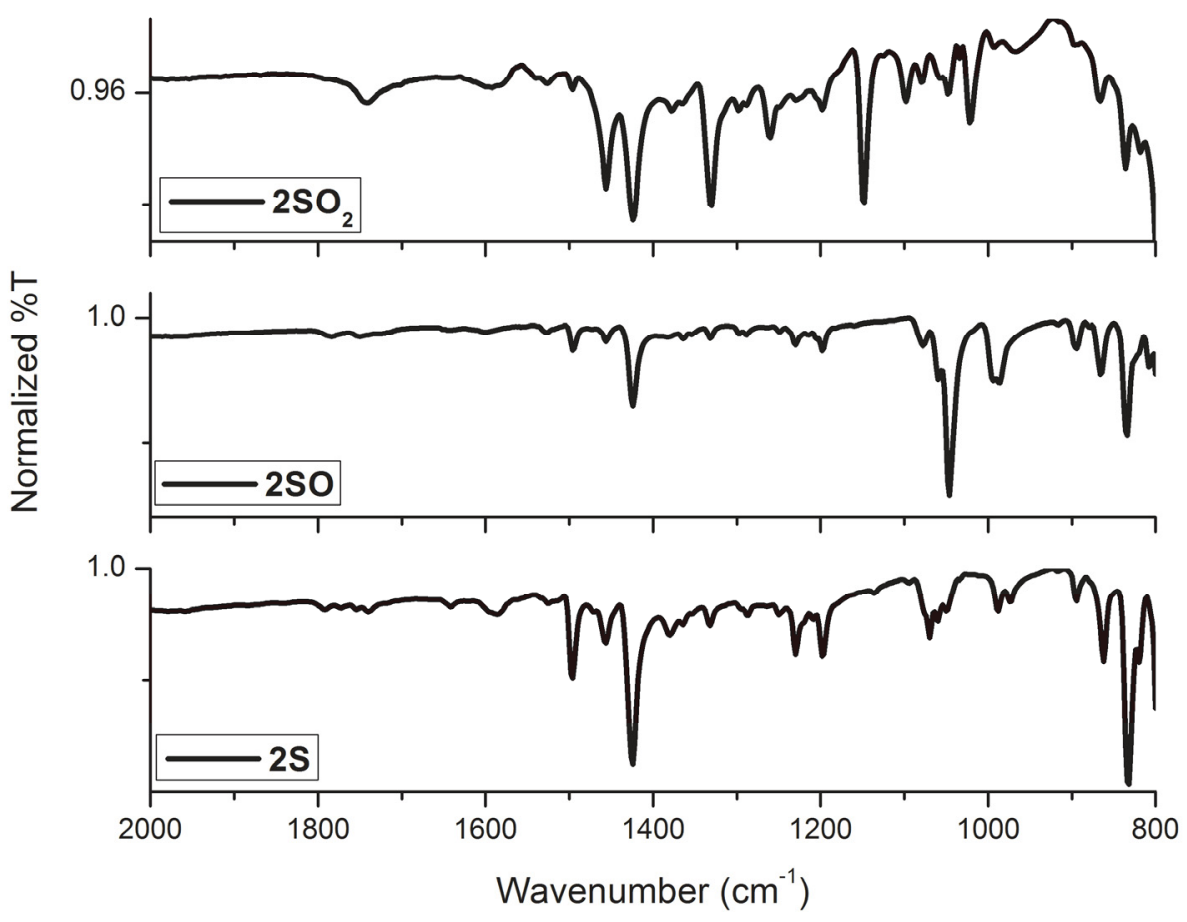

Figure S22. Infrared spectra of $\mathbf{2 S O}_{2}, 2 \mathrm{SO}$ and $2 \mathrm{~S}$. 

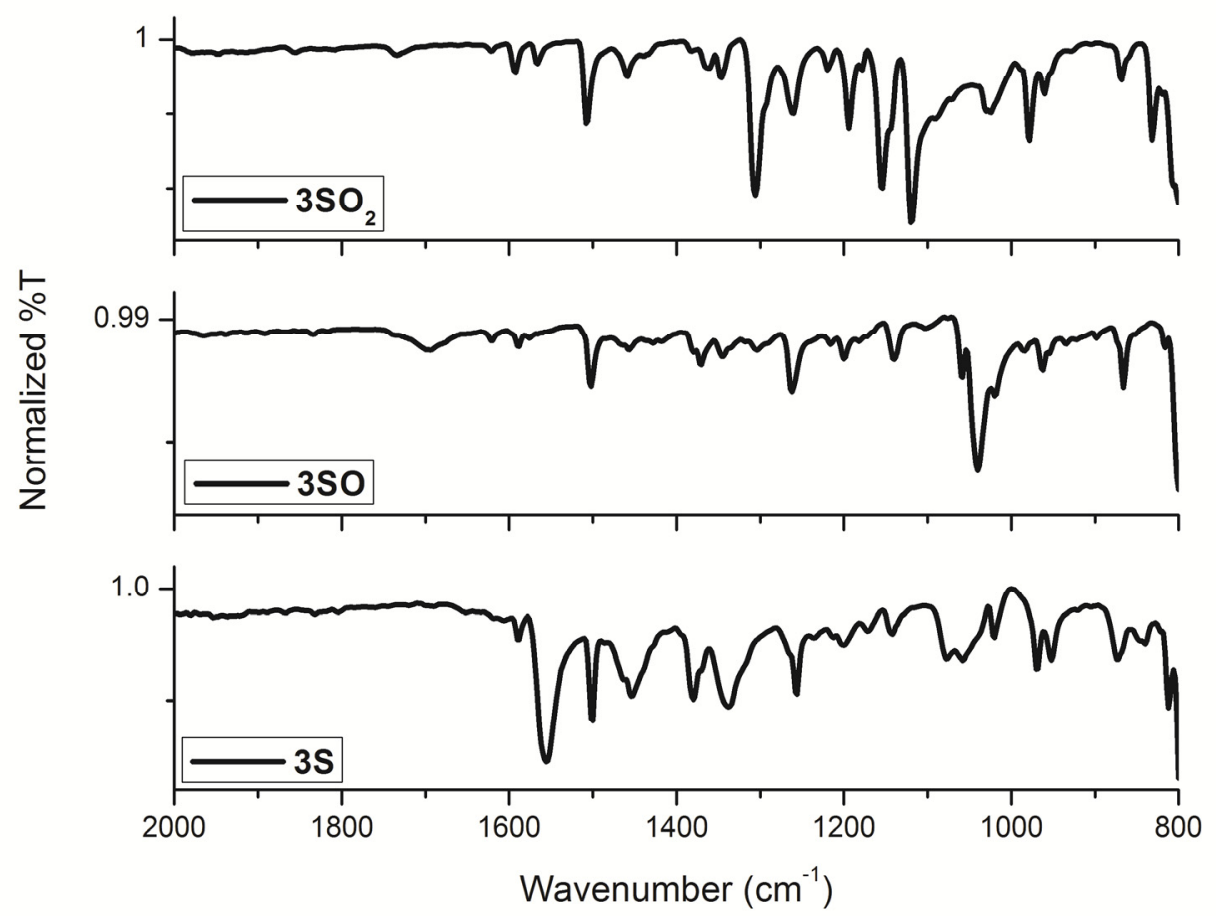

Figure S23. Infrared spectra of $\mathbf{3 S O}_{2}, \mathbf{3 S O}$ and $\mathbf{3 S}$.

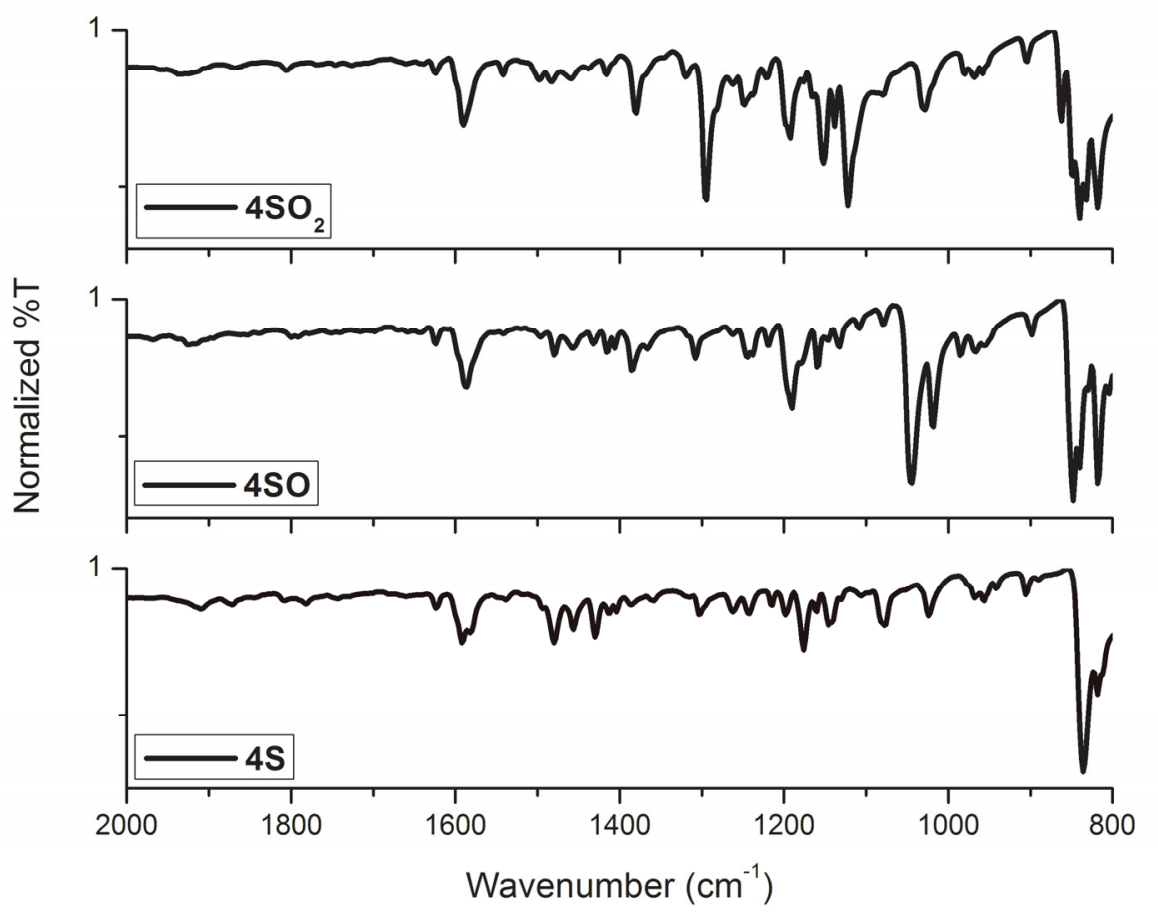

Figure S24. Infrared spectra of $4 \mathrm{SO}_{2}, 4 \mathrm{SO}$ and $4 \mathrm{~S}$. 

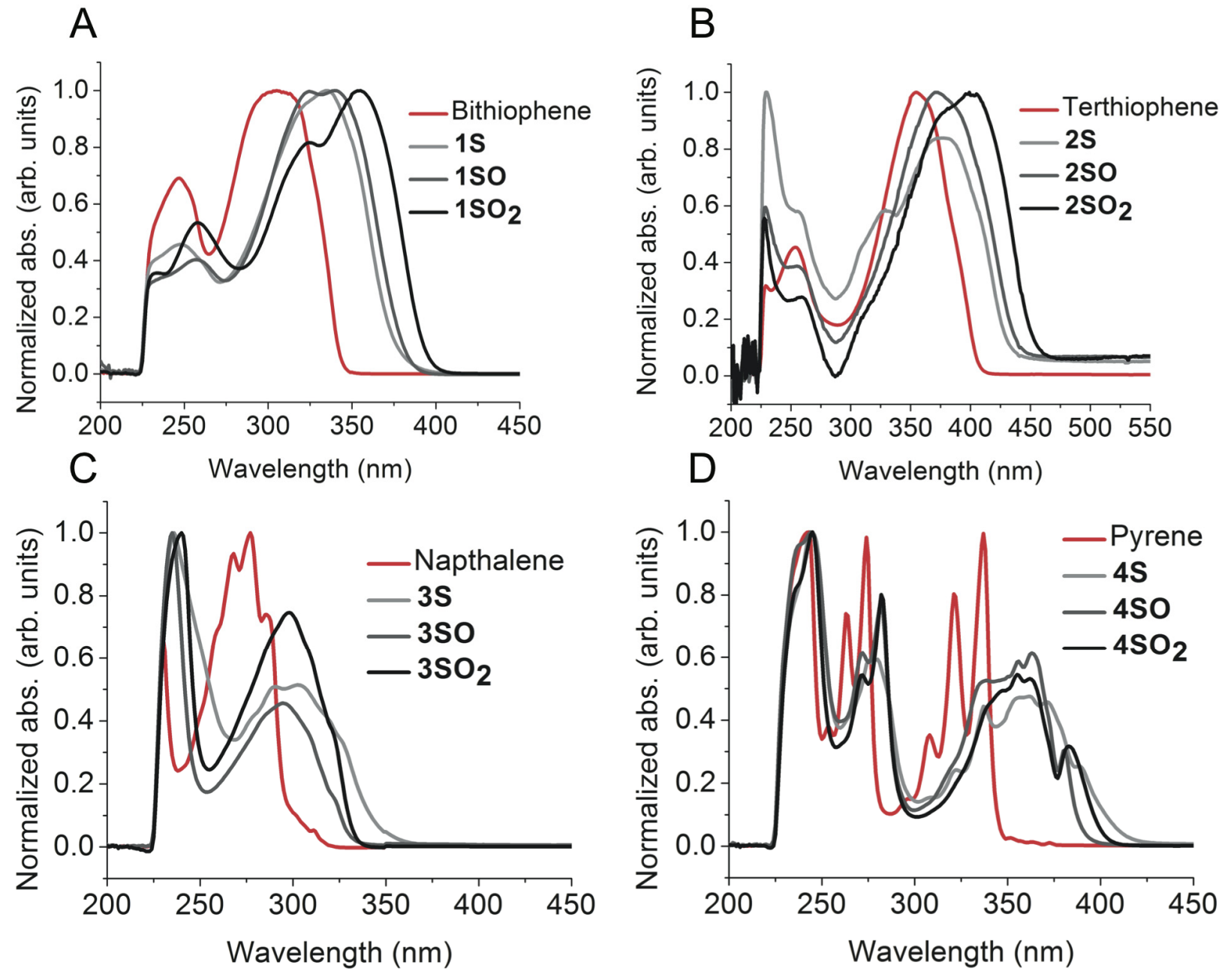

Figure S25. Relative UV-VIS spectra for (A) 1S, 1SO, 1SO (D) $4 \mathrm{~S}, 4 \mathrm{SO}, \mathrm{SSO}_{2}$. For comparison, the spectrum for each parent arene is also shown ( - ). 

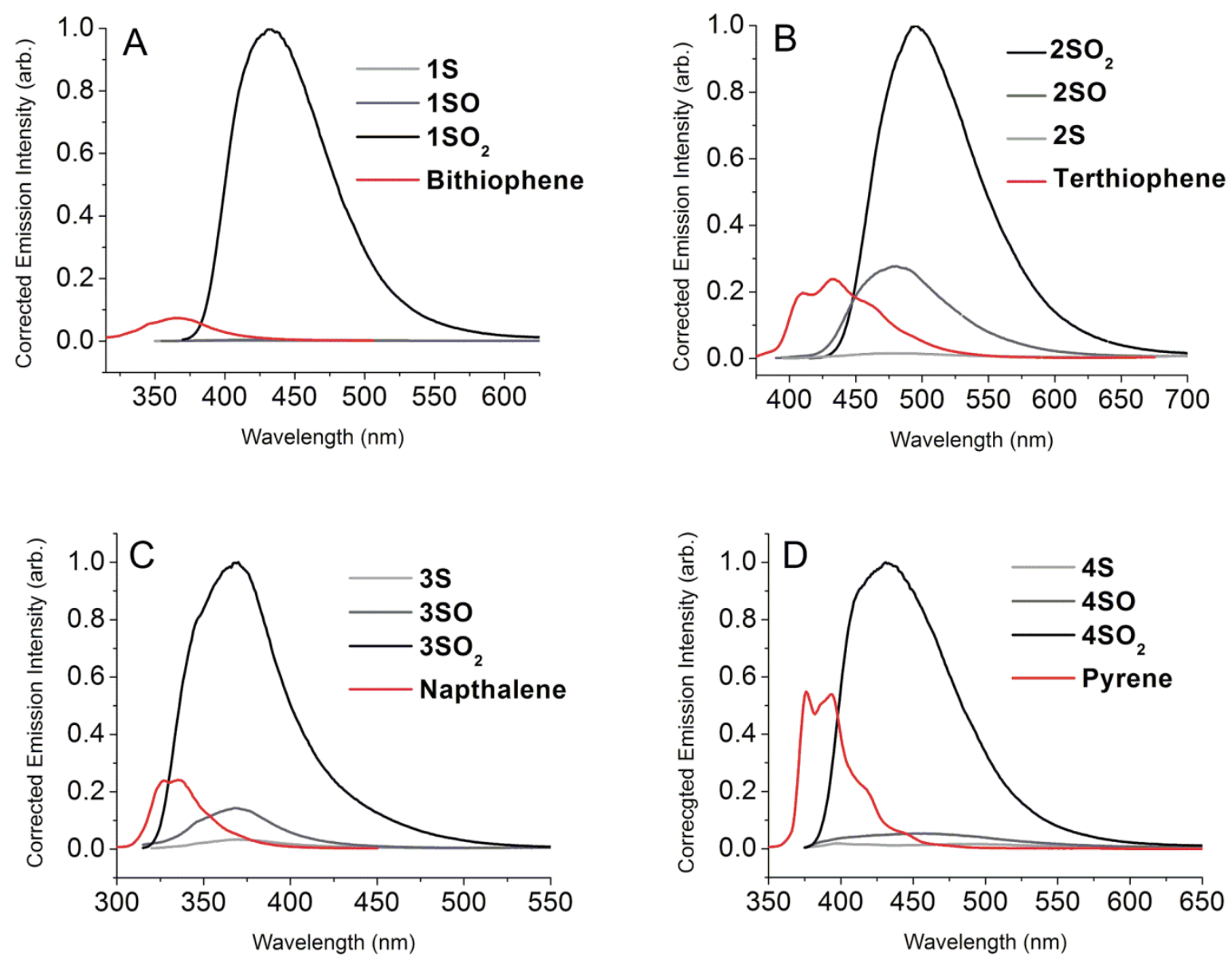

Figure S26. Absorbance corrected emission spectra for all compounds, including the parent arenes.

Table S7. Solid state $\Phi_{\mathrm{f}}$ values.

\begin{tabular}{|c|c|c|c|}
\hline Compound & $\Phi_{\mathrm{f}}$ & Compound & $\Phi_{\mathrm{f}}$ \\
\hline $\mathbf{1 S}$ & 0.02 & $\mathbf{2 S}$ & 0.07 \\
\hline $\mathbf{1 S O}$ & 0.00 & $\mathbf{2 S O}$ & 0.12 \\
\hline $\mathbf{1 S O}_{\mathbf{2}}$ & 0.18 & $\mathbf{2 S O}_{\mathbf{2}}$ & 0.08 \\
\hline
\end{tabular}




\section{References}

1. Boens, N. Qin, W.; Basarić, N.; Hofkens, J.; Ameloot, M.; Pouget, J.; Lefèvre, J.-P.; Valeur, B.; Gratton, E.; vande Ven, M; Silva, N.D., Jr.; Engelborghs, Y.; Willaert, K.; Sillen, A.; Rumbles, G.; Phillips, D.; Visser, A.J.W.G.; Malak, H.; Gryczynski, I.; Szabo, A.G.; Krajcarski, D.T.; Tamai, N.; Miura, A. Anal. Chem. 2007, 79, 2137-2149.

2. (a) Fonseca Guerra, C.; Snijders, J. G.; te Velde, G.; Baerends, E. J. Theor. Chem. Acc. 1998, 99, 391-403. (b) Fonseca Guerra, C.; Visser, O.; Snijders, J. G.; te Velde, G.;

Baerends, E. J., In: Methods and Techniques for Computational Chemistry; Clementi, E. and Corongiu, G., Eds.; STEF: Cagliari, 1995; p. 305-395. (c) Baerends, E. J.; Ellis, D. E.; Ros, P. Chem. Phys. 1973, 2, 41-51. (d) Boerrigter, P. M.; te Velde, G.; Baerends, E. J. Int. J. Quantum Chem. 1988, 33, 87-113. (e) te Velde, G.; Baerends, E. J. J. Comp. Phys. 1992, 99, 84-98. (f) Snijders, J. G.; Baerends, E. J.; Vernooijs, P. At. Nucl. Data Tables 1981, 26, 483-509. (g) Krijn, J.; Baerends, E. J. Fit Functions in the HFS Method; Internal Report (in Dutch); Vrije Universiteit: Amsterdam, 1984. (h) Slater, J. C. Quantum Theory of Molecules and Solids Vol. 4; McGraw-Hill: New York, 1974. (i) Vosko, S. H.; Wilk, L.; Nusair, M. Can. J. Phys. 1980, 58, 1200-1211. (j) Becke, A. D. J. Chem. Phys. 1986, 84, 4524-4529. (k) Becke, A. Phys. Rev. A 1988, 38, 3098-3100. (1) Perdew, J. P. Phys. Rev. B 1986, 33, 8822-8824 (Erratum: Phys. Rev. B 1986, 34, 7406). (m) Fan, L.; Ziegler, T. J. Chem. Phys. 1991, 94, 6057-6063. (n) Schipper, P. R. T.; Gritsenko, O. V.; van Gisbergen, S. J. A.; Baerends, E. .J. J. Chem. Phys. 2000, 112, 1344-1352. (o) Velde, G. T.;

Bickelhaupt, F. M.; Baerends, E. J.; Guerra, C. F.; Van Gisbergen, S. J. A.; Snijders, J. G.; Ziegler, T. J. Comput. Chem. 2001, 22, 931-967.

3. Martinie, R. J.; Bultema, M. N.; Vander Wal, M. N.; Burkhart, B. J.; vander Griend, D. A.; DeKock, R. L. J. Chem. Educ. 2011, 88, 1094-1097. Michalak, A.; DeKock, R. L.; Ziegler, T. J. Phys. Chem. A. 2008, 112, 7256-7263.

4. Fonseca Guerra, C.; Handgraaf, J.-W.; Baerends, E. J.; Bickelhaupt, F. M. J. Comput. Chem. 2003, 25, 189-210.

5. (a) Chang, C.; Pelissier, M.; Durand, P. Phys. Scr. 1986, 34, 394-404. (b) van Lenthe, E.; Baerends, E. J.; Snijders, J. G. J. Chem. Phys. 1993, 99, 4597-4610. (c) van Lenthe, E.; Baerends, E. J.; Snijders, J. G. J. Chem. Phys. 1994, 101, 9783-9792. (d) van Lenthe, E.; van Leeuwen, R.; Baerends, E. J.; Snijders, J. G. Int. J. Quantum Chem. 1996, 57, 281-293. (e) van Lenthe, E.; Ehlers, A.; Baerends, E. J. J. Chem. Phys. 1999, 110, 8943-8953. 